\title{
Homoclinic orbits to invariant tori near a homoclinic orbit to center-center-saddle equilibrium
}

\author{
Oksana Koltsova $^{1}$, Lev Lerman ${ }^{2}$, Amadeu Delshams $^{3}$, Pere Gutiérrez $^{3}$ \\ ${ }^{1}$ Dept. of Comput. Math. and Cybernetics, University of Nizhny Novgorod, \\ 23 Gagarin Ave., 603600 Nizhny Novgorod, Russia \\ ${ }^{2}$ Dept. of Diff. Equat., University of Nizhny Novgorod, \\ and Research Inst. for Applied Math. and Cybernetics, \\ 10 Ul'yanov St., 603600 Nizhny Novgorod, Russia \\ ${ }^{3}$ Dept. de Matem. Aplicada I, Universitat Politècnica de Catalunya, \\ Diagonal 647, 08028 Barcelona, Spain \\ koltsova@uic.nnov.ru, lermanl@mm.unn.ru, \\ amadeu.delshams@upc.es, pere.gutierrez@upc.es
}

\begin{abstract}
We consider a perturbation of an integrable Hamiltonian vector field with three degrees of freedom with a center-center-saddle equilibrium having a homoclinic orbit or loop. With the help of the Poincaré map, we study the homoclinic intersections between the stable and unstable manifolds associated to persistent hyperbolic KAM tori, on the center manifold near the equilibrium. If the perturbation is such that the homoclinic loop is preserved, we establish that, in general, the manifolds intersect along 8,12 or 16 transverse homoclinic orbits. On the other hand, in a more generic situation (the loop is not preserved) the manifolds intersect along 4 transverse homoclinic orbits, though a small neighborhood of the loop has to be excluded. In a first approximation, those homoclinic orbits can be detected as nondegenerate critical points of a Melnikov potential. We also develop an alternative Melnikov approximation in order to study the splitting of the loop itself.
\end{abstract}

\section{$1 \quad$ Introduction and set-up}

Consider on a smooth $\left(C^{\infty}\right)$ symplectic manifold $(M, \Omega)$ with smooth symplectic 2 -form $\Omega$ a 3 d.o.f. smooth Hamiltonian vector field $X_{H}$ with a near integrable Hamiltonian $H=$ $H_{0}+\varepsilon H_{1}$. Here $H_{0}$ defines an integrable vector field, that is, we assume that there are two additional smooth first integrals $F_{1}, F_{2}$ such that the triple $\left(H_{0}, F_{1}, F_{2}\right)$ forms an involutive set of functions (see, for instance, $[2,1]$ ), and the differentials of these three functions are independent on an open dense set in $M$. Our main assumptions for $X_{H_{0}}$ are the following. 
Assumption $1 X_{H_{0}}$ has a simple singular point $O$ of center-center-saddle type.

The latter means that

1. The spectrum of the linearization matrix $L$ of $X_{H_{0}}$ at $O$ consists of two pairs of pure imaginary eigenvalues $\pm i \omega_{1}, \pm i \omega_{2}$ and a pair of reals $\pm \lambda$, all of them nonzero.

2. The commuting matrices $L, M_{1}, M_{2}$, coming from the linearizations of the vector fields $X_{H_{0}}, X_{F_{1}}, X_{F_{2}}$ at $O$, are linearly independent over the complex numbers and generate a Cartan subalgebra, i.e., the linear span of $L, M_{1}, M_{2}$ with complex coefficients contains a diagonalizable matrix with different eigenvalues.

Under this assumption the Vey-Eliasson theorem [16, 5] ensures that in some neighborhood $U$ of $O$ there exist smooth symplectic coordinates $\left(x_{1}, x_{2}, x_{3}, y_{1}, y_{2}, y_{3}\right)$ (that is, $\left.\Omega=d x \wedge d y=\sum d x_{i} \wedge d y_{i}\right)$ such that the functions $H_{0}, F_{1}, F_{2}$ have the following representations

$$
H_{0}=h\left(\xi_{1}, \xi_{2}, \eta\right), F_{1}=f_{1}\left(\xi_{1}, \xi_{2}, \eta\right), F_{2}=f_{2}\left(\xi_{1}, \xi_{2}, \eta\right),
$$

with $h, f_{1}, f_{2}$ smooth and $\xi_{1}=\left(x_{1}^{2}+y_{1}^{2}\right) / 2, \xi_{2}=\left(x_{2}^{2}+y_{2}^{2}\right) / 2, \eta=x_{3} y_{3}$. This result for the analytic case was proved by Vey in [16] and was extended to the $C^{\infty}$-case by Eliasson [5].

The quoted result allows us to describe completely the structure of $X_{H_{0}}$ locally near $O$. The representation obtained permits to write down the integrals in the following form where, without loss of generality, we can suppose that all integrals vanish at $O$

$$
\begin{aligned}
& H_{0}=\omega_{1} \xi_{1}+\omega_{2} \xi_{2}+\lambda \eta+\cdots, \\
& F_{1}=\nu_{1} \xi_{1}+\nu_{2} \xi_{2}+\mu \eta+\cdots, \\
& F_{2}=\kappa_{1} \xi_{1}+\kappa_{2} \xi_{2}+\sigma \eta+\cdots,
\end{aligned}
$$

here dots mean terms of higher order in the variables $\left(\xi_{1}, \xi_{2}, \eta\right)$. In this form the simplicity condition for the point $O$ is equivalent to the condition

$$
\Delta=\left|\begin{array}{lll}
\omega_{1} & \omega_{2} & \lambda \\
\nu_{1} & \nu_{2} & \mu \\
\kappa_{1} & \kappa_{2} & \sigma
\end{array}\right| \neq 0 .
$$

Below, without loss of generality, we regard $\lambda$ to be positive and, for definiteness, suppose $\omega_{1}>0$. In Vey-Eliasson coordinates all local invariant manifolds through $O$ can be easily characterized. In particular, the local one-dimensional stable manifold $W^{s}$ is the axis $x_{3}$, the local one-dimensional unstable manifold $W^{u}$ is the axis $y_{3}$, the local 4-dimensional symplectic center manifold $W^{c}$ is the disk $x_{3}=y_{3}=0$, the local 5-dimensional center-stable manifold $W^{c s}$ is $y_{3}=0$ and the center-unstable manifold $W^{c u}$ is $x_{3}=0$.

Our next assumption concerns the orbit behavior on $W^{c}$. The restriction of $X_{H_{0}}$ on $W^{c}$ gives a 2 d.o.f Hamiltonian vector field with an elliptic singular point at $O$. This vector field is integrable, and the restriction of any of $F_{1}, F_{2}$ gives an additional integral. Moreover, since $O$ is simple, it will also be simple for the vector field on $W^{c}$, since if $\Delta \neq 0$, then one of the determinants of the second order composed from the first and second columns in $\Delta$ does not vanish. 
Assumption 2 The restriction of $X_{H_{0}}$ on $W^{c}$ has $O$ as a singular point of general elliptic type (that is, nondegenerate or isoenergetically nondegenerate).

Let $\left.H_{0}\right|_{W^{c}}=\omega_{1} \xi_{1}+\omega_{2} \xi_{2}+A \xi_{1}^{2}+2 B \xi_{1} \xi_{2}+C \xi_{2}^{2}+\cdots$ be the restriction of $X_{H_{0}}$ on $W^{c}$. Recall that the condition for an elliptic singular point to be of the general elliptic type requires that some quantities calculated in terms of the fourth order in the normal form do not vanish. For our coordinates it gives either

$$
\left|\begin{array}{ll}
A & B \\
B & C
\end{array}\right| \neq 0 \text { (nondegenerate) or }\left|\begin{array}{ccc}
A & B & \omega_{1} \\
B & C & \omega_{2} \\
\omega_{1} & \omega_{2} & 0
\end{array}\right| \neq 0 \text { (isoenergetically nondegenerate). }
$$

Thus we see that if the restriction of $X_{H_{0}}$ on $W^{s}$ gives a point of general elliptic type, then point this persists for a perturbed vector field on its local center manifold.

This ensures the existence of invariant 2-dimensional KAM tori under the perturbation of $H_{0}$ on the related persistent center submanifold [6]. They become hyperbolic tori for the whole 3 d.o.f. Hamiltonian.

Our third assumption (see below) is usually fulfilled for an integrable system with a (unique) singular point of the type under consideration. It concerns the behavior of the global stable and unstable manifolds. We assume that they coalesce forming a homoclinic loop. Indeed, locally near $O$, the set $W^{u}$ consists of three orbits: $O$ and two semi-orbits which tend to $O$ when $t \rightarrow-\infty$. Suppose that the continuation in $t$ for positive time of one of these orbits stays in a compact domain of the phase space $M$. Then, it can be shown $[9,8]$ that, under some mild assumptions on the behavior of the integrable vector field $X_{H_{0}}$, this orbit has to return into a neighborhood of $O$ and then should tend to $O$ when $t \rightarrow \infty$, forming a homoclinic orbit to $O$ (its closure is frequently called a homoclinic loop $\Gamma$ ). We assume that this is the case, namely

Assumption $3 X_{H_{0}}$ has a homoclinic loop $\Gamma$ to $O$.

Remark 1 The same as in assumption 3 is true for the second semi-orbit in $W^{u}$, so usually in an integrable system without discrete symmetries and with a compact Hamiltonian level of the point $O$, two outgoing semi-orbits merge with two ingoing semi-orbits forming the "figure eight" set.

Let us summarize the contributions of this paper, which are concerned with the behavior of the perturbed Hamiltonian $H=H_{0}+\varepsilon H_{1}$ on a neighborhood of one loop in the phase space.

We start, in Section 2, by studying the behavior of the integrable Hamilonian $H_{0}$ near the loop $\Gamma$. To this end, we choose four local sections to orbits in $W^{s}$ and $W^{u}$, defined by the equalities

$$
N_{ \pm}^{s}=\left\{x_{3}= \pm d\right\}, \quad N_{ \pm}^{u}=\left\{y_{3}= \pm d\right\},
$$

respectively, and we describe the map from $N_{ \pm}^{s}$ to $N_{ \pm}^{u}$ (local map) defined by the flow. After that we discuss the form of the map from $N_{ \pm}^{u}$ to $N_{ \pm}^{s}$ defined by the flow along the global 
piece of the homoclinic orbit (global map). Their composition gives us the Poincaré map near the homoclinic orbit.

In Section 3, we consider the local map for the perturbed Hamiltonian. With the help of the center manifold theory and KAM theory, we introduce new symplectic coordinates such that the persistent hyperbolic invariant tori (on the perturbed center manifold near the equilibrium), as well as their stable and unstable manifolds, become flat.

We consider in Section 4 the perturbed global map and study the existence of homoclinic intersections between the stable and unstable manifolds associated to persistent hyperbolic tori. If the perturbation is such that the homoclinic loop is preserved, we show that, for a wide set of tori, the manifolds intersect along 8, 12 or 16 transverse homoclinic orbits (Theorem 1). On the other hand, in a more generic situation in which the loop is not preserved, the manifolds intersect along 4 transverse homoclinic orbits (Theorem 2).

In Section 5 we use the Poincaré-Melnikov method and prove that a first approximation for the splitting distance between the invariant manifolds can be expressed in terms of a Melnikov potential (Theorem 3). Then, the transverse homoclinic orbits can be detected as nondegenerate critical points of the Melnikov potential, and this allows us to obtain the results on the existence of such orbits in a more precise formulation (Theorems 4 and 5). In concrete examples, the number of homoclinic orbits can be found by computing the Melnikov potential explicitly.

It has to be pointed out that, for the validity of the Poincaré-Melnikov method, a small neighborhood of the loop has to be excluded. To overcome this, in Section 6 we develop an alternative Melnikov approximation in order to study the splitting of the loop itself (Theorem 6).

\section{$1.1 \quad$ Some examples}

We end this introduction with some concrete examples of integrable Hamiltonians for which the previous assumptions are fulfilled. In symplectic (global) coordinates $\left(x_{1}, y_{1}, x_{2}, y_{2}, p, q\right)$, consider

$$
\begin{aligned}
& H_{0}=h_{0}\left(\xi_{1}, \xi_{2}\right)+\frac{p^{2}}{2}+V(q), \\
& h_{0}\left(\xi_{1}, \xi_{2}\right)=\omega_{1} \xi_{1}+\omega_{2} \xi_{2}+A \xi_{1}^{2}+2 B \xi_{1} \xi_{2}+C \xi_{2}^{2}+\cdots,
\end{aligned}
$$

with $A, B, C$ as required in assumption 2, and a given potential $V(q)$. This potential is assumed to have a nondegenerate maximum at $q=0$; say $V(0)=V^{\prime}(0)=0, V^{\prime \prime}(0)=-1$. Next, we provide some examples; the associated homoclinic loops are parameterized by $\left(0,0,0,0, \dot{q}_{0}(t), q_{0}(t)\right)$ with $q_{0}(t)$ as given below.

1. Pendulum: $V(q)=\cos q-1, q \in \mathbb{T} ; q_{0}(t)= \pm 4 \arctan e^{t}$.

2. Duffing: $V(q)=-q^{2} / 2+q^{4} / 4, q \in \mathbb{R} ; q_{0}(t)= \pm \sqrt{2} \operatorname{sech} t$.

3. Cubic: $V(q)=-q^{2} / 2+q^{3} / 3, q \in \mathbb{R} ; q_{0}(t)=\frac{3}{2} \operatorname{sech}^{2} \frac{t}{2}$. 
We point out that in examples 1 and 2 there are two symmetric homoclinic loops; instead, in example 3 there is only one loop (the second semi-orbit is not contained in a compact set).

In those examples, the symplectic (local) coordinates leading to the representations (1) are given by the well-known transformation to Birkhoff normal form for a 1-degree-of-freedom Hamiltonian near a hyperbolic equilibrium point [12]. First, the linear change

$$
\left(\begin{array}{l}
p \\
q
\end{array}\right)=\frac{1}{\sqrt{2}}\left(\begin{array}{cc}
1 & 1 \\
-1 & 1
\end{array}\right)\left(\begin{array}{l}
\tilde{x}_{3} \\
\tilde{y}_{3}
\end{array}\right)
$$

takes, in the three cases, the term $p^{2} / 2+V(q)$ in $H_{0}$ into

$$
\tilde{x}_{3} \tilde{y}_{3}+\left\{\begin{array}{l}
\frac{1}{96}\left(\tilde{y}_{3}-\tilde{x}_{3}\right)^{4}-\cdots, \\
\frac{1}{16}\left(\tilde{y}_{3}-\tilde{x}_{3}\right)^{4} \\
\frac{1}{6 \sqrt{2}}\left(\tilde{y}_{3}-\tilde{x}_{3}\right)^{3} .
\end{array}\right.
$$

Then, a symplectic change $\left(\tilde{x}_{3}, \tilde{y}_{3}\right)=\left(x_{3}, y_{3}\right)+\mathcal{O}_{2}\left(x_{3}, y_{3}\right)$ with a convergent expansion in a neighborhood leads to a function of $\eta=x_{3} y_{3}$. Note that $\lambda=1$ in the three examples. In all three examples we may take the first integrals $F_{1}=\xi_{1}, F_{2}=\xi_{2}$. So, locally, we have as in $(1)$

$$
\begin{aligned}
& H_{0}=\omega_{1} \xi_{1}+\omega_{2} \xi_{2}+\eta+\cdots, \\
& F_{1}=\xi_{1}, F_{2}=\xi_{2}, \text { and } \Delta=1 \neq 0 .
\end{aligned}
$$

Thus, $O$ is a simple singular point for the integrable system.

\section{The Poincaré map for the integrable system}

First of all, we will distinguish two possibilities for the Hamiltonian $H_{0}$ :

1. $\omega_{1} \omega_{2}>0$ (definite case);

2. $\omega_{1} \omega_{2}<0$ (indefinite case).

These two cases have different foliation into levels $H=c$ on the center manifold and in a neighborhood of homoclinic orbit $\Gamma$. Indeed, the restriction of $X_{H_{0}}$ onto $W^{c}$ gives an integrable system with two degrees of freedom having a simple elliptic point at $O$. Its local orbit structure is easily seen from the representation (1) (see also [9]). For the definite case, due to our choice $\omega_{1}>0$, we get $\omega_{2}>0$ as well. Here each level $H_{0}=c, c>0$, on $W^{c}$ is a 3-sphere for $c$ small enough, in this sphere the system has two linked periodic orbits, one from the symplectic disk $D_{1}=\left\{x_{3}=y_{3}=x_{2}=y_{2}=0\right\}$ and another one from the symplectic disk $D_{2}=\left\{x_{3}=y_{3}=x_{1}=y_{1}=0\right\}$. Other orbits in this level belong to a one-parameter family of invariant tori foliating the sphere. The singular level $H_{0}=0$ consists of only point $O$. Thus, for the definite case all tori in $W^{c}$ lie in levels $H_{0}>0$.

For the indefinite case $\omega_{1} \omega_{2}<0$ the singular level $H_{0}=0$ in $W^{c}$ is topologically a cone over a 2 -torus ${ }^{1}$. Each level $H_{0}=c, c \neq 0$, in $W^{c}$ is topologically (and differentially) manifold

\footnotetext{
${ }^{1}$ This means that one takes the direct product $\mathbb{T}^{2} \times I, I=[0,1]$, and $\mathbb{T}^{2} \times\{0\}$ is identified to the point.
} 
$D^{2} \times S^{1}$ (solid tori), whose center circle is a periodic orbit from one $D_{i}$-disk, other orbits belong to invariant tori.

The local map preserves the integrals $\xi_{1}, \xi_{2}, \eta$, as well as $H_{0}, F_{1}, F_{2}$ being functions of $\xi_{1}, \xi_{2}, \eta$ with nondegenerate Jacobian $D\left(H_{0}, F_{1}, F_{2}\right) / D\left(\xi_{1}, \xi_{2}, \eta\right)$. Let us write down the Hamilton equations

$$
\begin{aligned}
& \dot{x}_{i}=-\frac{\partial h}{\partial \xi_{i}} y_{i}, \quad \dot{y}_{i}=\frac{\partial h}{\partial \xi_{i}} x_{i}, \quad i=1,2, \\
& \dot{x}_{3}=-\frac{\partial h}{\partial \eta} x_{3}, \quad \dot{y}_{3}=\frac{\partial h}{\partial \eta} y_{3} .
\end{aligned}
$$

Since the function $h$ and its derivatives depend only on $\xi_{1}, \xi_{2}, \eta$ and are constant along a given orbit, we can integrate (3) as linear equations with constant coefficients and get the local flow

$$
\begin{aligned}
& x_{i}(t)=x_{i}^{0} \cos \left(t h_{\xi_{i}}^{0}\right)-y_{i}^{0} \sin \left(t h_{\xi_{i}}^{0}\right), \quad y_{i}(t)=x_{i}^{0} \sin \left(t h_{\xi_{i}}^{0}\right)+y_{i}^{0} \cos \left(t h_{\xi_{i}}^{0}\right), \quad i=1,2, \\
& x_{3}(t)=x_{3}^{0} \exp \left[-t h_{\eta}^{0}\right], \quad y_{3}(t)=y_{3}^{0} \exp \left[t h_{\eta}^{0}\right]
\end{aligned}
$$

where lower indices by $h$ mean derivatives with respect to related variables, and upper zeroes mark that the calculations in $h$ are made at $\xi_{i}^{0}=\left(\left(x_{i}^{0}\right)^{2}+\left(y_{i}^{0}\right)^{2}\right) / 2, i=1,2$, and $\eta^{0}=x_{3}^{0} y_{3}^{0}$.

Thus, we see that the signs of the variables $x_{3}$ and $y_{3}$ are preserved by the local flow. The local map on both $N_{ \pm}^{s}$ is disconnected, the discontinuity takes place along the trace of $W^{c s}\left(y_{3}=0\right)$. So the part of $N_{+}^{s}$ where $y_{3}>0$ is transformed onto $N_{+}^{u}$, and the part with $y_{3}<0$ is transformed onto $N_{-}^{u}$. The same is true for $N_{-}^{s}$.

First we solve the equation $h\left(\xi_{1}, \xi_{2}, \eta\right)=c$ in $U$. Since $h(0,0,0)=0$ and $h_{\eta}(0,0,0)=$ $\lambda \neq 0$, this equation can be solved with respect to $\eta$ for all values of variables in $U$ (may be in some smaller neighborhood of $O$ which we will denote also $U$, as well),

$$
\eta=a\left(\xi_{1}, \xi_{2}, c\right)=\frac{1}{\lambda}\left(c-\omega_{1} \xi_{1}-\omega_{2} \xi_{2}-\cdots\right),
$$

$a(0,0,0)=0$. We may always regard $N_{ \pm}^{s}, N_{ \pm}^{u}$ belonging to $U$, then using inequality $\partial a / \partial c \neq$ 0 in $U$, we can introduce coordinates $\left(x_{1}, y_{1}, x_{2}, y_{2}, c\right)$ in $N_{ \pm}^{s}$ (and $N_{ \pm}^{u}$ ) instead of coordinates $\left(x_{1}, y_{1}, x_{2}, y_{2}, y_{3}\right)\left(\left(x_{1}, y_{1}, x_{2}, y_{2}, x_{3}\right)\right.$, respectively) by formulae $y_{3}=a\left(\xi_{1}, \xi_{2}, c\right) / d$ in $N_{ \pm}^{s}$, and similarly in $N_{ \pm}^{u}: x_{3}=a\left(\xi_{1}, \xi_{2}, c\right) / d$. On each 4-disk $H_{0}=c$ in $N_{+}^{s}$, coordinates $\left(x_{1}, y_{1}, x_{2}, y_{2}\right)$ are symplectic with respect to the standard symplectic 2-form $d x_{1} \wedge d y_{1}+d x_{2} \wedge d y_{2}$, being the restriction of $\Omega$ to this disk.

Now we describe how the trace of the stable manifold of a given torus in $W^{c}$ is represented in $N_{ \pm}^{s}$ in Vey-Eliasson coordinates, and the same for the trace of the unstable manifold in $N_{ \pm}^{u}$. The union of stable manifolds of all tori in $W^{c}$ make up $W^{c s}$ itself, it is given by the equation $y_{3}=0$, the same is true for the union of unstable manifolds making up $W^{c u}$ given as $x_{3}=0$. The restrictions of $H_{0}$ on $W^{c s}$ and $W^{c u}$ in these coordinates have the same representations, given by putting $\eta=0$ in $H_{0}$. To fix a definite torus $T_{0}^{2}$ in $W^{c}$ one should assign definite values to the functions $\xi_{1}=\xi_{1}^{0}, \xi_{2}=\xi_{2}^{0}$ and put $\eta=0$. In coordinates $\left(x_{1}, y_{1}, x_{2}, y_{2}, c\right)$ in 
$N_{ \pm}^{s}$ the trace of $W^{s}\left(T_{0}^{2}\right)$ is given as $\xi_{1}=\xi_{1}^{0}, \xi_{2}=\xi_{2}^{0}, c=h\left(\xi_{1}^{0}, \xi_{2}^{0}, 0\right)$. The same set $c, \xi_{1}^{0}, \xi_{2}^{0}$ determines the trace of $W^{u}\left(T_{0}^{2}\right)$ in $N_{ \pm}^{u}$.

To write down the form of the local map, let us integrate equations (3) and find the passage time by orbits from $N_{ \pm}^{s}$ till $N_{ \pm}^{u}$. From formulae (4) for solutions we immediately derived that the passage time from $N_{ \pm}^{s}$ till $N_{ \pm}^{u}$ is equal to

$$
t_{p}=\frac{1}{h_{\eta}^{0}} \ln \frac{d}{\left|y_{3}^{0}\right|} .
$$

Plugging this into the first four equations in (4) we get the local map $T_{0}$. The simplest form of this map is obtained in the symplectic polar coordinates $\left(\xi_{1}, \xi_{2}, \varphi_{1}, \varphi_{2}\right)$ on $N_{ \pm}^{u}(c)=$ $N_{ \pm}^{u} \cap\left\{H_{0}=c\right\}$, and the same with zeroth upper indices on $N_{ \pm}^{s}(c)=N_{ \pm}^{s} \cap\left\{H_{0}=c\right\}$, where $x_{i}=\sqrt{2 \xi_{i}} \cos \varphi_{i}, \quad y_{i}=\sqrt{2 \xi_{i}} \sin \varphi_{i}$ :

$$
\xi_{i}^{1}=\xi_{i}^{0}, \quad \varphi_{i}^{1}=\varphi_{i}^{0}-\frac{\partial a\left(\xi_{1}^{0}, \xi_{2}^{0}, c\right)}{\partial \xi_{i}^{0}} \ln \frac{d^{2}}{a\left(\xi_{1}^{0}, \xi_{2}^{0}, c\right)} \quad(\bmod 2 \pi) .
$$

These polar coordinates degenerate on the planes $x_{1}=y_{1}=0$ and $x_{2}=y_{2}=0$, respectively, but these planes $x_{1}^{0}=y_{1}^{0}=0$ and $x_{2}^{0}=y_{2}^{0}=0$ are transformed by the local flow onto planes $x_{1}^{1}=y_{1}^{1}=0$ and $x_{2}^{1}=y_{2}^{1}=0$, respectively, so their traces on $N_{ \pm}^{s}$ and $N_{ \pm}^{u}$ are mapped into each other.

To study the global map we suppose, without loss of generality, that the outgoing local piece of $\Gamma$ in $U$ coincides with the semiaxis $y_{3}>0$.

Global map $S_{0}: N_{+}^{u} \rightarrow N_{ \pm}^{s}$ is defined by the flow of the vector field $X_{H_{0}}$ near a global piece of $\Gamma$. Along orbits of $X_{H_{0}}$ the values of $H_{0}, F_{1}, F_{2}$ are preserved. Inside $U$ quadratic functions $\xi_{1}, \xi_{2}, \eta$ are also integrals and they are smooth functions of $H_{0}, F_{1}, F_{2}$, due to the condition $D\left(H_{0}, F_{1}, F_{2}\right) / D\left(\xi_{1}, \xi_{2}, \eta\right) \neq 0$ in $U$. It implies that if at a point in $N_{+}^{u}$ the functions $\xi_{1}, \xi_{2}, \eta$ take definite values, then at the image of this point in $N_{+}^{s}$ (or $N_{-}^{s}$ ) the values of these functions are the same, since the values of functions the $H_{0}, F_{1}, F_{2}$ are preserved.

We stress that the choice of the sign in $N_{ \pm}^{s}$ depends on which cross-section of two ones $\Gamma$ returns to. In the examples considered in Section 1.1, it is not hard to check that $\Gamma$ returns to $N_{-}^{s}$ in example 1 , and to $N_{+}^{s}$ in examples 2 and 3 .

For a fixed $c$ the form of the global map in coordinates $\left(\xi_{1}, \xi_{2}, \varphi_{1}, \varphi_{2}\right)$ is defined by the preservation of coordinates $\xi_{1}, \xi_{2}$; that is, a torus $\xi_{1}=\xi_{1}^{0}, \xi_{2}=\xi_{2}^{0}$ in $N_{+}^{u}(c)$ is transformed onto the torus in $N_{ \pm}^{s}(c)$ with the same values of $\xi_{1}, \xi_{2}$, and the only thing we need to determine is the form of the map in $\varphi_{i}, i=1,2$. Since disks $\xi_{1}=0$ and $\xi_{2}=0$ are transformed to disks $\bar{\xi}_{1}=0$ and $\bar{\xi}_{2}=0$, respectively, then, due to the smoothness of the global map, and its symplecticity, the map in $\varphi_{i}$ is of the form

$$
\bar{\varphi}_{1}=\varphi_{1}+\frac{\partial R}{\partial \xi_{1}}(\bmod 2 \pi), \quad \bar{\varphi}_{2}=\varphi_{2}+\frac{\partial R}{\partial \xi_{2}}(\bmod 2 \pi),
$$

where $R=R\left(\xi_{1}, \xi_{2}, c\right)$. Thus, the global map $S_{0}: N_{+}^{u}(c) \longrightarrow N_{ \pm}^{s}(c)$ is close to a fixed rotation of angles

$$
\gamma_{i}=\left.\frac{\partial R}{\partial \xi_{i}}\right|_{\xi=0}
$$


It will be convenient to change the coordinates in $N_{+}^{u}(c)$ in such a way that this map becomes close to the identity. To do this, with a rotation of coordinates $X=\left(x_{1}, x_{2}, y_{1}, y_{2}\right)^{\top}$ we introduce new coordinates $Y=\left(u_{1}, u_{2}, v_{1}, v_{2}\right)^{\top}$ in $N_{+}^{u}(c)$ :

$$
\begin{aligned}
& u_{i}=x_{i} \cos \gamma_{i}-y_{i} \sin \gamma_{i}, \\
& v_{i}=x_{i} \sin \gamma_{i}+y_{i} \cos \gamma_{i}, \quad i=1,2 .
\end{aligned}
$$

For the images in $N_{ \pm}^{s}(c)$, we keep the coordinates $\bar{X}=\left(\bar{x}_{1}, \bar{x}_{2}, \bar{y}_{1}, \bar{y}_{2}\right)^{\top}$. Then, the global map takes the form:

$$
\begin{gathered}
\bar{x}_{i}=u_{i} \cos \frac{\partial R_{1}}{\partial \xi_{i}}-v_{i} \sin \frac{\partial R_{1}}{\partial \xi_{i}}, \\
\bar{y}_{i}=u_{i} \sin \frac{\partial R_{1}}{\partial \xi_{i}}+v_{i} \cos \frac{\partial R_{1}}{\partial \xi_{i}}
\end{gathered}
$$

where $R_{1}=R\left(\xi_{1}, \xi_{2}, c\right)-\gamma_{1} \xi_{1}-\gamma_{2} \xi_{2},\left.\frac{\partial R_{1}}{\partial \xi_{i}}\right|_{\xi=0}=0$. Expanding the right hand side of $(6-7)$ at $\xi=0$, the global map $S_{0}$ casts as follows

$$
\bar{X}=Y+F(Y, c),
$$

where the function $F$ begins with third order terms in $Y$.

Now we are able to study properties of Poincaré map which is the composition of local and global maps, $S_{0} \circ T_{0}$. We consider first the definite case $\omega_{1} \omega_{2}>0$, that is, both $\omega_{i}>0$. $\Gamma$ returns after global travel either on $N_{-}^{s}$ (case $\mathbf{A}$ ) or on $N_{+}^{s}$ (case $\left.\mathbf{B}\right)$. The difference between these two possibilities consists in the different topology of the level $H_{0}=0$. For the case A the disk $y_{3}=0$ (the trace of $W^{c s}$ ) in $N_{-}^{s}$ touches the disk $H_{0}=0$ at only one point $\xi_{1}=\xi_{2}=0$, for other points of this level $y_{3}>0$, and so, all these points are transformed by the local map to $N_{+}^{u}$. Therefore, the local map has a discontinuity only at the trace of $\Gamma$ on this level, but can be determined by continuity up to a homeomorphism of the disk. In particular, a neighborhood of $\Gamma$ in the level $H_{0}=0$ is homeomorphic to $D^{4} \times S^{1}$.

For $c>0$ the intersection of disks $H_{0}=c$ and $W^{c s}$ (i.e., $y_{3}=0$ ) in $N_{-}^{s}$ is a 3 -sphere $\Sigma_{c}$ given by solutions of the equation $h\left(\xi_{1}, \xi_{2}, 0\right)=c$. Points inside $\Sigma_{c}$ are transformed to $N_{-}^{u}$, and points outside $\Sigma_{c}$ (but inside of some larger sphere defined by the size of $U$ ) are transformed to $N_{+}^{u}$, any transverse to $\Sigma_{c}$ segment through the point on $\Sigma_{c}$ breaks. These considerations define a domain of the Poincaré map on $N_{-}^{s}$.

For $c<0$ all points of some 4-disk centered at the origin in $N_{-}^{s}$ are smoothly mapped by the local map $T_{0}$ onto some 4 -disk in $N_{+}^{u}$. Thus we get

Lemma 1 Suppose $\omega_{1} \omega_{2}>0$. Then for the case $A$ and $c=0$ the domain of the Poincaré map is a punctured 4-disk. For $c<0$ the domain is some 4-disk, and for $c>0$ the domain is a layer between two concentric spheres.

For the case $\mathbf{B}$ ( $\Gamma$ returns to $N_{+}^{s}$ ) the situation is opposite. Namely, for $c=0$ all points (in $N_{+}^{s}$ ) excepting the trace of $\Gamma$ in 4-disk $H_{0}=0$, are mapped by $T_{0}$ onto $N_{-}^{u}$ and lost (i.e. go out from the neighborhood under consideration). If $c>0$, then the points inside of the 
small sphere $\Sigma_{c}$ determined by $y_{3}=0$ are mapped onto $N_{+}^{u}$, the points out of $\Sigma_{c}$ are lost. For $c<0$ all points of the 4-disk are lost.

In the indefinite case $\omega_{1} \omega_{2}<0$, the situaton is more complicated. To fix ideas, suppose that $\Gamma$ returns to $N_{+}^{s}$. As was said before, the local map breaks at the points of the trace of $W^{c s}$ on $N_{+}^{u}$ (they are given for different $c$ by the equation $h\left(\xi_{1}, \xi_{2}, 0\right)=c$ or $\left.y_{3}=a\left(\xi_{1}, \xi_{2}, c\right) / d=0\right)$. These traces are described for different $c$ as follows. If $c=0$, then this trace in 4-disk $N_{+}^{s}(0)$ is diffeomorphic to a cone over torus. This set divides this 4-disk into two regions, $a\left(\xi_{1}, \xi_{2}, 0\right)$ is positive in one region (these points are transformed by the local map to $N_{+}^{u}$ ) and negative in another one (these points are transformed by the local map to $N_{-}^{u}$ and are not considered further, since $\Gamma$ intersects $N_{+}^{u}$ ). If $c \neq 0$, the situation is the same as for $c=0$, only the trace of $W^{c s}$ given by $a=0$ here is smooth $D^{3}$, it also divides 4 -disk $H_{0}=c$ into regions with opposite signs of $a$, so only one of these domains is mapped to $N_{+}^{u}$.

For the problem about homoclinic orbits to invariant tori in $W^{c}$ we only need to know how images under action of the global map of traces of unstable manifolds of tori from $W^{c}$ in $N_{+}^{u}$ intersect traces of their stable manifolds in $N_{ \pm}^{s}$.

Thus, we have got that for integrable system all invariant tori in $W^{c}$ have their stable and unstable manifolds coinciding, that is all orbits are homoclinic. As we will see later, in general for a perturbed system only a finite number of homoclinic orbits to invariant torus survive.

\section{The perturbed local map}

Now let us consider a perturbed Hamiltonian system with Hamiltonian $H=H_{0}+\varepsilon H_{1}$, where $H_{0}$, as above, is integrable with a homoclinic orbit $\Gamma$ to a center-center-saddle singular point $O$. Such the singular point persists under a perturbation and smoothly depends on $\varepsilon$. Without loss of generality one can assume that this singular point does not move under the perturbation. This will be assumed henceforth.

Using Vey-Eliasson coordinates in some neighborhood of $O$ one can consider the restriction of the perturbed system to $U$. Then the system will be a perturbation of system (3). We take the same two cross-sections $N_{+}^{s}$ (or $N_{-}^{s}$ ), $N_{+}^{u}$ as for the integrable system. These cross-sections are determined by the integrable system therefore we omit lower indices \pm . The local map $T_{\varepsilon}: N^{s} \rightarrow N^{u}$ is a perturbation of the map (5). Similarly, we get the perturbation of the global map $S_{\varepsilon}: N^{u} \rightarrow N^{s}$. We need to analyse the properties of these two maps.

We begin with the study of the local map. The principal difference with the integrable case consists in that, due to KAM theory, local center manifold fails to be filled with invariant 2-tori: only a Cantor set of a positive measure is filled with tori. Stable and unstable manifolds of these tori survived cut $N^{s}$ and $N^{u}$, respectively, giving related Cantor sets of tori on them. For our purposes we use two known results: the theorem on the persistence of local center-stable and center-unstable manifolds [3, 7], and the KAM theorem by Pöschel [13].

For $\varepsilon=0$ the vector field has center-stable manifold $W^{c s}: y_{3}=0$, center-unstable mani- 
fold $W^{c u}: x_{3}=0$, and center manifold $W^{c}: x_{3}=y_{3}=0$. They are all flat, that is, pieces of the related coordinate subspaces. First what we do is the flattenning of the corresponding perturbed manifolds $W_{\varepsilon}^{c s}, W_{\varepsilon}^{c u}, W_{\varepsilon}^{c}$. Recall that, by the center manifold theory [3, 7], these manifolds exist and are $C^{r}$-smooth (with arbitrary high $r$, if the neighborhood of $O$ where these manifolds are defined is chosen sufficiently small). In the coordinates we use these manifolds can be represented as graphs of the following $C^{r}$-functions: $y_{3}=f\left(x_{1}, y_{1}, x_{2}, y_{2}, x_{3}, \varepsilon\right)$ $\left(\right.$ for $\left.W^{c s}\right), x_{3}=g\left(x_{1}, y_{1}, x_{2}, y_{2}, y_{3}, \varepsilon\right)$ (for $\left.W^{c u}\right),\left(x_{3}, y_{3}\right)=w\left(x_{1}, y_{1}, x_{2}, y_{2}, \varepsilon\right)$ (for $\left.W^{c}\right)$. Onedimensional stable and unstable manifolds also persist, they stay as smooth as the perturbation.

As was said before, we assume that $\lambda>0$ and $\omega_{1}>0$. The spectrum of the linearized vector field at $O$ is $\left(-\lambda(\varepsilon), \pm i \omega_{1}(\varepsilon), \pm i \omega_{2}(\varepsilon), \lambda(\varepsilon)\right)$. Thus, there are invariant linear subspaces of the tangent space at $O$ for eigenvalues $\left(-\lambda(\varepsilon), \pm i \omega_{1}(\varepsilon), \pm i \omega_{2}(\varepsilon)\right.$ ) (it is the tangent space to $W_{\varepsilon}^{c s}$ at $O$ ), the same for $\left( \pm i \omega_{1}(\varepsilon), \pm i \omega_{2}(\varepsilon), \lambda(\varepsilon)\right.$ ) (it is the tangent space to $W_{\varepsilon}^{c u}$ at $O$ ) and $\left( \pm i \omega_{1}(\varepsilon), \pm i \omega_{2}(\varepsilon)\right)$ (tangent space to $W_{\varepsilon}^{c}$ at $O$ ). Making previously the linear change of variables one can suppose that the related linear invariant subspaces coincide with the coordinate planes. Then, the functions $f, g, w$ begin with the terms of the second order in their variables. We assume this below.

To flatten related manifolds we use the following assertion.

Proposition 1 There is a canonical change of variables

$$
\left(x_{1}, y_{1}, x_{2}, y_{2}, x_{3}, y_{3}\right) \rightarrow\left(X_{1}, Y_{1}, X_{2}, Y_{2}, X_{3}, Y_{3}\right)
$$

such that the Hamiltonian takes the form

$$
\begin{aligned}
& H\left(X_{1}, Y_{1}, X_{2}, Y_{2}, X_{3}, Y_{3}, \varepsilon\right)=\mathcal{H}_{1}\left(X_{1}, Y_{1}, X_{2}, Y_{2}, \varepsilon\right)+ \\
& X_{3} Y_{3} \mathcal{H}_{2}\left(X_{1}, Y_{1}, X_{2}, Y_{2}, X_{3}, Y_{3}, \varepsilon\right), \quad \mathcal{H}_{2}(0,0,0,0,0,0)=\lambda(\varepsilon),
\end{aligned}
$$

where the expansion of $\mathcal{H}_{1}\left(X_{1}, Y_{1}, X_{2}, Y_{2}, \varepsilon\right)$ in spatial variables begins with $\omega_{1}(\varepsilon)\left(X_{1}^{2}+\right.$ $\left.Y_{1}^{2}\right) / 2+\omega_{2}(\varepsilon)\left(X_{2}^{2}+Y_{2}^{2}\right) / 2$.

Proof. We only make one step, namely we will flatten $W^{c s}$. One can use a standard result from symplectic geometry (see, for instance, [10]) but here we are able to give an easy direct proof. The idea is the same as for proving the Darboux theorem in [2]. Let $y_{3}=f\left(x_{1}, y_{1}, x_{2}, y_{2}, x_{3}, \varepsilon\right)$ be the representation of $W^{c s}$. Introduce the variable $Y_{3}=$ $y_{3}-f\left(x_{1}, y_{1}, x_{2}, y_{2}, x_{3}, \varepsilon\right)$ (recall that $f$ is of the order 2 in spatial variables). Take $Y_{3}$ as a Hamilton function and consider the related Hamiltonian vector field. The hypersurface $x_{3}=g\left(x_{1}, y_{1}, x_{2}, y_{2}, y_{3}, \varepsilon\right)$ is transversal to the flow near $O$, since at this point $\dot{x}_{3}=-1$. Take as the conjugated coordinate to $Y_{3}$ the function $X_{3}\left(x_{1}, y_{1}, x_{2}, y_{2}, x_{3}, y_{3}, \varepsilon\right)$ defined as the time of reaching the point $\left(x_{1}, y_{1}, x_{2}, y_{2}, x_{3}, y_{3}\right)$ for an orbit starting on the hypersurface $x_{3}=g$. It is evident that $X_{3}=0$ on this hypersurface. The Poisson bracket of these functions satisfies $\left\{X_{3}, Y_{3}\right\} \equiv 1$. Therefore, the functions $X_{3}, Y_{3}$ are independent and commute. The common level $X_{3}=Y_{3}=0$ is a smooth symplectic 4-disk $D$. One can take a symplectic chart $\left(X_{1}, Y_{1}, X_{2}, Y_{2}\right)$ at the point $O$ in $D$. Observe that the commuting independent functions $X_{3}, Y_{3}$ generate a Poisson action of the group $\mathbb{R}^{2}$, with two-dimensional orbits being 
transversal to $D$. This means that one can extend Darboux coordinate functions given in $D$ to some neighborhood of $D$ in transverse direction obtaining the complete set of functions giving Darboux coordinates near $O$ (see details in [2]). In these coordinates, due to the construction, $W^{c s}$ is given by the equation $Y_{3}=0$, as desired.

Thus, after these transformations we get the Hamiltonian in the form we sought. The corresponding differential system casts as follows

$$
\begin{aligned}
& \dot{X}_{1}=-\frac{\partial \mathcal{H}_{1}}{\partial Y_{1}}-X_{3} Y_{3} \frac{\partial \mathcal{H}_{2}}{\partial Y_{1}}, \quad \dot{Y}_{1}=\frac{\partial \mathcal{H}_{1}}{\partial X_{1}}+X_{3} Y_{3} \frac{\partial \mathcal{H}_{2}}{\partial X_{1}} \\
& \dot{X}_{2}=-\frac{\partial \mathcal{H}_{1}}{\partial Y_{2}}-X_{3} Y_{3} \frac{\partial \mathcal{H}_{2}}{\partial Y_{2}}, \quad \dot{Y}_{2}=\frac{\partial \mathcal{H}_{1}}{\partial X_{2}}+X_{3} Y_{3} \frac{\partial \mathcal{H}_{2}}{\partial X_{2}} \\
& \dot{X}_{3}=-X_{3} \mathcal{H}_{2}-X_{3} Y_{3} \frac{\partial \mathcal{H}_{2}}{\partial Y_{3}}, \quad \dot{Y}_{3}=Y_{3} \mathcal{H}_{2}+X_{3} Y_{3} \frac{\partial \mathcal{H}_{2}}{\partial X_{3}}
\end{aligned}
$$

Next we apply the KAM results of Pöschel [13] to the restriction of the system to the center manifold $X_{3}=Y_{3}=0$ : there is a smooth symplectic change of variables $\left(X_{1}, Y_{1}, X_{2}, Y_{2}\right) \rightarrow\left(I_{1}, \theta_{1}, I_{2}, \theta_{2}\right)$ smoothly depending on $\varepsilon$ such that in new coordinates Hamiltonian $\mathcal{H}_{1}$ turns into $\hat{\mathcal{H}}_{1}=h_{1}\left(I_{1}, \theta_{1}, I_{2}, \theta_{2}, \varepsilon\right)$, where the derivatives

$$
\frac{\partial h_{1}}{\partial \theta_{1}}, \frac{\partial h_{1}}{\partial \theta_{2}}
$$

vanish on a Cantorian set of $\left(I_{1}, I_{2}\right)$ of positive measure near the origin, that is, at these tori we get $\hat{\mathcal{H}}_{1}=h_{1}\left(I_{1}, I_{2}\right)$. In [13] it is required the necessary smoothness to be greater than $3 n-1$, where $n$ is the number of degrees of freedom. For our case, we have $n=2$ since the KAM result is applied on the center manifold. This necessary smoothness can be reached in a sufficiently small neighborhood of $O$.

We stress that the new coordinates $\left(I_{1}, \theta_{1}, I_{2}, \theta_{2}\right)$ can be taken $\mathcal{O}(\varepsilon)$-close to the polar coordinates (5).

In the KAM coordinates $\left(I_{1}, \theta_{1}, I_{2}, \theta_{2}, X_{3}, Y_{3}\right)$, the differential system turns into

$$
\begin{array}{ll}
\dot{I}_{1}=-\frac{\partial h_{1}}{\partial \theta_{1}}-X_{3} Y_{3} \frac{\partial \mathcal{H}_{2}}{\partial \theta_{1}}, & \dot{\theta}_{1}=\frac{\partial h_{1}}{\partial I_{1}}+X_{3} Y_{3} \frac{\partial \mathcal{H}_{2}}{\partial I_{1}} \\
\dot{I}_{2}=-\frac{\partial h_{1}}{\partial \theta_{2}}-X_{3} Y_{3} \frac{\partial \mathcal{H}_{2}}{\partial \theta_{2}}, & \dot{\theta}_{2}=\frac{\partial h_{1}}{\partial I_{2}}+X_{3} Y_{3} \frac{\partial \mathcal{H}_{2}}{\partial I_{2}} \\
\dot{X}_{3}=-X_{3} \mathcal{H}_{2}-X_{3} Y_{3} \frac{\partial \mathcal{H}_{2}}{\partial Y_{3}}, & \dot{Y}_{3}=Y_{3} \mathcal{H}_{2}+X_{3} Y_{3} \frac{\partial \mathcal{H}_{2}}{\partial X_{3}} .
\end{array}
$$

Let us consider a torus from the Cantorian set in $W^{c}$. From (9), setting $X_{3}=Y_{3}=0$ we get $\dot{I}_{1}=0, \dot{I}_{2}=0$. Thus, we see that $Y_{3}=0, I_{1}=I_{1}^{0}, I_{2}=I_{2}^{0}$ define the stable manifold of a preserved torus $X_{3}=Y_{3}=0, \quad I_{1}=I_{1}^{0}, \quad I_{2}=I_{2}^{0}$. The same is valid for the unstable manifold of such a torus, one only needs to replace $Y_{3}$ by $X_{3}$. 
So, in order to find homoclinic orbits to the torus preserved, one needs to prove that the image under the global mapping $S_{\varepsilon}$ of the trace on $N^{u}$ of local unstable manifold of this torus intersects the trace of local stable manifold on $N^{s}$ of the same torus.

\section{The perturbed global map}

Under a perturbation, in general, the Hamiltonian system loses integrability. In particular, it can lead to the splitting of homoclinic loop to the center-center-saddle $O$. Nevertheless, there are perturbations which preserve the homoclinic loop, that is, perturbed stable and unstable manifolds of the singular point coalesce. These perturbations belong to a codimension 4 submanifold near the integrable Hamiltonian $H_{0}$ in the space of all smooth Hamiltonians with $C^{r}$-topology, $r$ is greater than $3 n-1$ (with $n=2$ ). Indeed, the local stable and unstable manifolds are one-dimensional, their traces $m_{s}$ on $N^{s}$ and $n_{u}$ on $N^{u}$, respectively, are points on the 4-disks $N^{s} \cap\{H=H(O)\}$ and $N^{u} \cap\{H=H(O)\}$. The perturbation moves these traces on disks. The global map transforms $n_{u}$ to a point $m_{u}$ on $N^{s}$. The distance between $m_{s}$ and $m_{u}$ characterizes the splitting of the loop. It is clear that in order to make related points $m_{s}$ and $m_{u}$ coincident one has to have at least a 4-parametric unfolding of the initial Hamiltonian. Thus, codimension of Hamiltonians with homoclinic loops to centercenter-saddle is equal to four. This can be proved rigorously but we omit it here (see also Section 6 for a related result). Let us denote the set of Hamiltonians with loops near $H_{0}$ in the space of all $C^{r}$-Hamiltonians as $\mathcal{L}$.

The perturbed global map has the form of a perturbation of (8). So we get

$$
\bar{X}=Y+F(Y, c)+\varepsilon G(Y, c, \varepsilon) .
$$

Recall that $Y=0$ corresponds to the trace of $\Gamma$ on $N^{u}$. Let us expand function $G$ in $Y$ at $Y=0$ up to third order terms. The linear term in $Y$ takes the form $(E+\varepsilon U(\varepsilon, c)) Y$ with symplectic matrix $E+\varepsilon U$. Therefore this close to identity matrix can be written as the exponent of some Hamiltonian matrix $I A$ with a symmetric $A$ (we denote $I$ the standard symplectic matrix). Thus

$$
\bar{X}=\varepsilon \alpha(\varepsilon, c)+\exp [\varepsilon I A(\varepsilon, c)] Y+F(Y, c)+\varepsilon G_{1}(Y, c, \varepsilon),
$$

where the expansion of function $G_{1}$ begins with the second order terms in $Y$.

The case of unfolding lying in $\mathcal{L}$ is characterized by the condition

$$
\alpha(\varepsilon, 0) \equiv 0 \text {. }
$$

Theorem 1 For the case $(\mathbf{L})$ and $\varepsilon$ small enough there exists a region $V$ in the positive quadrant of the plane $\left(I_{1}, I_{2}\right)$ near the point $(0,0)$ such that the perturbed system has either 8, 12 or 16 transverse (on the related level of the Hamiltonian) homoclinic orbits to every perturbed persistent Diophantine tori $I_{1}=I_{1}^{0}, I_{2}=I_{2}^{0},\left(I_{1}^{0}, I_{2}^{0}\right) \in V$, on the center manifold $W_{\varepsilon}^{c}$. 
Remark 2 The region $V$ consists of the part of the quadrant $I_{1}>0, I_{2}>0$ where the function $R$ defined in (14) is a Morse function, i.e. all its critical points are nondegenerate. This means that $V$ is the whole quadrant except for small neighborhoods of some rays $I_{1} / I_{2}=$ const. In fact, this nondegeneracy condition for $R$ can be interpreted as a condition on the first order deviation in $\varepsilon$ for the global map at the trace of $\Gamma$ on $N^{u}(0)$. In Section 5, this deviation is written in terms of the Melnikov potential.

Proof. Let us fix a torus in $W_{\varepsilon}^{c}: I_{1}=I_{1}^{0}, I_{2}=I_{2}^{0}$, in the Cantorian set of persistent tori. Traces of its stable and unstable manifolds on $N^{s}(c)$ and $N^{u}(c)$, as was shown above, have the same representation $T_{s}^{2}=\left\{\bar{I}_{1}=I_{1}^{0}, \bar{I}_{2}=I_{2}^{0}\right\}, T_{u}^{2}=\left\{I_{1}=I_{1}^{0}, I_{2}=I_{2}^{0}\right\}$. Let us find $T_{s}^{2} \cap S_{\varepsilon}\left(T_{u}^{2}\right)$. To this end, square both sides of the every equality in (10) and sum up separately left hand sides and right hand sides for symplectically conjugated coordinates

$$
\begin{aligned}
& \frac{1}{2}\left(\bar{x}_{1}^{2}+\bar{y}_{1}^{2}\right)=\frac{1}{2}\left(u_{1}^{2}+v_{1}^{2}\right)+\varepsilon\left(A_{1}(Y, c)+\varepsilon B_{1}(Y, \varepsilon, c)\right), \\
& \frac{1}{2}\left(\bar{x}_{2}^{2}+\bar{y}_{2}^{2}\right)=\frac{1}{2}\left(u_{2}^{2}+v_{2}^{2}\right)+\varepsilon\left(A_{2}(Y, c)+\varepsilon B_{2}(Y, \varepsilon, c)\right),
\end{aligned}
$$

where

$$
\begin{aligned}
A_{1}(Y, c)= & u_{1}\left(\alpha_{1}(0, c)-a_{13} u_{1}-a_{23} u_{2}-a_{33} v_{1}-a_{34} v_{2}\right)+ \\
& v_{1}\left(\alpha_{3}(0, c)+a_{11} u_{1}+a_{12} u_{2}+a_{13} v_{1}+a_{14} v_{2}\right)+O\left(\|Y\|^{3}\right), \\
A_{2}(Y, c)= & u_{2}\left(\alpha_{2}(0, c)-a_{14} u_{1}-a_{24} u_{2}-a_{34} v_{1}-a_{44} v_{2}\right)+ \\
& v_{2}\left(\alpha_{4}(0, c)+a_{12} u_{1}+a_{22} u_{2}+a_{23} v_{1}+a_{24} v_{2}\right)+O\left(\|Y\|^{3}\right) .
\end{aligned}
$$

To find the intersection $T_{s}^{2}$ and $S_{\varepsilon}\left(T_{u}^{2}\right)$ we set in the equalities obtained $\bar{I}_{i}=I_{i}=I_{i}^{0}$, $I_{i}=\left(u_{i}^{2}+v_{i}^{2}\right) / 2, i=1,2$. Thus we get

$$
\begin{gathered}
\varepsilon\left(A_{1}(Y, c)+\varepsilon B_{1}(Y, \varepsilon, c)\right)=0, \\
\varepsilon\left(A_{2}(Y, c)+\varepsilon B_{2}(Y, \varepsilon, c)\right)=0,
\end{gathered}
$$

Divide both sides of the equations (11) by $\varepsilon$ and take the limit $\varepsilon \rightarrow 0$. After introducing symplectic polar coordinates $u_{i}=\sqrt{2 I_{i}^{0}} \cos \theta_{i}, v_{i}=\sqrt{2 I_{i}^{0}} \sin \theta_{i}$, the system takes the form

$$
\begin{aligned}
2 \sqrt{I_{1}^{0} I_{2}^{0}}\left(a_{12} \sin \theta_{1} \cos \theta_{2}+\quad\right. & \left.a_{14} \sin \theta_{1} \sin \theta_{2}-a_{23} \cos \theta_{1} \cos \theta_{2}-a_{34} \cos \theta_{1} \sin \theta_{2}\right)+ \\
& \sqrt{2 I_{1}^{0}}\left(\alpha_{1}(0, c) \cos \theta_{1}+\alpha_{3}(0, c) \sin \theta_{1}\right)+ \\
& I_{1}^{0}\left(\left(a_{11}-a_{33}\right) \sin 2 \theta_{1}-2 a_{13} \cos 2 \theta_{1}\right)+O\left(\left\|I^{0}\right\|^{3 / 2}\right)=0, \\
2 \sqrt{I_{1}^{0} I_{2}^{0}}\left(a_{12} \cos \theta_{1} \sin \theta_{2}+\quad\right. & \left.a_{23} \sin \theta_{1} \sin \theta_{2}-a_{14} \cos \theta_{1} \cos \theta_{2}-a_{34} \sin \theta_{1} \cos \theta_{2}\right)+ \\
& \sqrt{2 I_{2}^{0}}\left(\alpha_{2}(0, c) \cos \theta_{2}+\alpha_{4}(0, c) \sin \theta_{2}\right)+ \\
& I_{2}^{0}\left(\left(a_{22}-a_{44}\right) \sin 2 \theta_{2}-2 a_{24} \cos 2 \theta_{2}\right)+O\left(\left\|I^{0}\right\|^{3 / 2}\right)=0 .
\end{aligned}
$$

For the case $(\mathbf{L})$, the homoclinic orbit to $O$, persists so $\alpha_{i}(\varepsilon, 0) \equiv 0$, therefore, $\alpha_{i}(0, c)=$ $c \bar{\alpha}_{i}(0, c)$. Since we have fixed the persistent torus, then in local coordinates the restriction of the Hamiltonian on $W_{\varepsilon}^{c}$ depends on $I_{1}^{0}, I_{2}^{0}$ only, that is, $c=h_{1}\left(I_{1}^{0}, I_{2}^{0}\right)=\omega_{1} I_{1}^{0}+\omega_{2} I_{2}^{0}+$ 
$O\left(\left\|I^{0}\right\|^{2}\right)$. Therefore, the terms containing $\alpha_{i}(0, c)$ can be included into $O\left(\left\|I^{0}\right\|^{3 / 2}\right)$ terms. Divide both sides of the equations by $\sqrt{I_{1}^{0} I_{2}^{0}}$ and denote $r=\sqrt{I_{1}^{0} / I_{2}^{0}}$. As a result system (12) takes the form

$$
\begin{aligned}
& r\left(\left(a_{11}-a_{33}\right) \sin 2 \theta_{1}-2 a_{13} \cos 2 \theta_{1}\right)+2\left(a_{12} \sin \theta_{1} \cos \theta_{2}+a_{14} \sin \theta_{1} \sin \theta_{2}-\right. \\
& \left.a_{23} \cos \theta_{1} \cos \theta_{2}-a_{34} \cos \theta_{1} \sin \theta_{2}\right)+O\left(\left\|I^{0}\right\|^{1 / 2}\right)=0, \\
& \frac{1}{r}\left(\left(a_{22}-a_{44}\right) \sin 2 \theta_{2}-2 a_{24} \cos 2 \theta_{2}\right)+2\left(a_{12} \cos \theta_{1} \sin \theta_{2}+a_{23} \sin \theta_{1} \sin \theta_{2}-\right. \\
& \left.a_{14} \cos \theta_{1} \cos \theta_{2}-a_{34} \sin \theta_{1} \cos \theta_{2}\right)+O\left(\left\|I^{0}\right\|^{1 / 2}\right)=0 .
\end{aligned}
$$

Let us fix $r$ and tend $I_{1}^{0} \rightarrow 0$ and $I_{2}^{0} \rightarrow 0$ along a ray $I_{1}^{0}=r^{2} I_{2}^{0}$ in the plane $\left(I_{1}, I_{2}\right)$. The equations obtained are written as

$$
\nabla R=0
$$

with

$$
\begin{aligned}
R= & -r\left(\frac{a_{11}-a_{33}}{2} \cos 2 \theta_{1}+a_{13} \sin 2 \theta_{1}\right)-\frac{1}{r}\left(\frac{a_{22}-a_{44}}{2} \cos 2 \theta_{2}+a_{24} \sin 2 \theta_{2}\right) \\
& -2\left(a_{12} \cos \theta_{1} \cos \theta_{2}+a_{23} \sin \theta_{1} \cos \theta_{2}+a_{14} \cos \theta_{1} \sin \theta_{2}+a_{34} \sin \theta_{1} \sin \theta_{2}\right) \\
= & -r\left(\frac{a_{11}-a_{33}}{2} \cos 2 \theta_{1}+a_{13} \sin 2 \theta_{1}\right)-\frac{1}{r}\left(\frac{a_{22}-a_{44}}{2} \cos 2 \theta_{2}+a_{24} \sin 2 \theta_{2}\right) \\
& -\left(\left(a_{12}-a_{34}\right) \cos \left(\theta_{1}+\theta_{2}\right)+\left(a_{23}+a_{14}\right) \sin \left(\theta_{1}+\theta_{2}\right)\right. \\
& \left.\quad+\left(a_{12}+a_{34}\right) \cos \left(\theta_{1}-\theta_{2}\right)+\left(a_{23}-a_{14}\right) \sin \left(\theta_{1}-\theta_{2}\right)\right) \\
= & s_{1} \cos \left(2 \theta_{1}-\sigma_{1}\right)+s_{2} \cos \left(\theta_{1}+\theta_{2}-\sigma_{2}\right) \\
& +s_{3} \cos \left(\theta_{1}-\theta_{2}-\sigma_{3}\right)+s_{4} \cos \left(2 \theta_{2}-\sigma_{4}\right),
\end{aligned}
$$

where the (non-negative) coefficients $s_{i}$ depend on $r$, and the phases $\sigma_{i}$ do not depend on $r$. It is clear that this gradient form of the system of equations is a direct consequence of the property that tori $T_{s}^{2}$ and $S_{\varepsilon}\left(T_{u}^{2}\right)$ are Lagrangian.

We are seeking nondegenerate critical points of function $R\left(\theta_{1}, \theta_{2}, r\right)$ at a fixed $r$ on the torus $\left(\theta_{1}, \theta_{2}\right)(\bmod 2 \pi)$. The nondegeneracy at a critical point $p$ is equivalent to the inequality

$$
\operatorname{det} \frac{\partial^{2} R}{\partial \theta^{2}}(p) \neq 0
$$

where $\theta=\left(\theta_{1}, \theta_{2}\right)$.

Let us observe that the function $R$ depends only on $\theta_{1}, \theta_{2}$ and $r$. This means that for all tori on the ray $I_{1}^{0}=r^{2} I_{2}^{0}$ this function is the same. In particular, if we have found a torus $I_{1}=I_{1}^{0}, I_{2}=I_{2}^{0}$ such that all critical points of $R$ on this torus are nondegenerate, then the same is true for all tori on this ray and for rays (i.e. $r$ ) sufficiently close to this one.

Applying Lemma 2 (see below) to the function $R(\cdot, \cdot, r)$ we see that, if all its critical points are nondegenerate, the number of such critical points must be 8,12 or 16 . Now let us restrict $r, 0<r_{0}<r<r_{1}$. If, for a fixed $r_{*}$ in this interval, the function $R$ has only nondegenerate critical points, then the same is true for some open interval of $r$ containing 
$r_{*}$. Therefore, the set $\mathcal{C}$ of all $r$ such that the function $R$ has only nondegenerate critical points is open and belongs to the interval $\left(r_{0}, r_{1}\right)$. For $r$ belonging to the boundary of $\mathcal{C}$, the function $R$ has at least one degenerate critical point.

Let us choose a compact set in $\mathcal{C}$ (for instance, a collection of a finite number of segments). Then for these $r \in \mathcal{C}$ the implicit function theorem can be applied to system (11), expressed in polar coordinates and divided by $\varepsilon$, uniformly in $r$ giving solutions $\theta_{i}\left(I_{1}^{0}, I_{2}^{0}, \varepsilon\right), i=1,2$, for all $\left(I_{1}^{0}, I_{2}^{0}, \varepsilon\right)$ close enough to $(0,0,0)$. The union of all rays with $r \in \mathcal{C}$ gives us the set $V$ of the theorem.

Finally, we select any torus $I=I_{1}^{0}, I=I_{2}^{0}$ in $W^{c}$ of the integrable system with a diophantine set of frequencies which persists for $0 \leq \varepsilon<\varepsilon_{0}$. If these $I_{1}^{0}, I_{2}^{0}$ such that $r=\sqrt{I_{1}^{0} / I_{2}^{0}} \in \mathcal{C}$ then we can obtain intersection on $N^{s}(c)$ of the stable and unstable manifolds of this torus $\left(\theta_{1}\left(I_{1}^{0}, I_{2}^{0}, \varepsilon\right), \theta_{2}\left(I_{1}^{0}, I_{2}^{0}, \varepsilon\right), c=h\left(I_{1}^{0}, I_{2}^{0}\right)\right)$.

Lemma 2 Let $R\left(\theta_{1}, \theta_{2}\right)$ be a scalar function on $\mathbb{T}^{2}$ of the form

$$
R=s_{1} \cos \left(2 \theta_{1}-\sigma_{1}\right)+s_{2} \cos \left(\theta_{1}+\theta_{2}-\sigma_{2}\right)+s_{3} \cos \left(\theta_{1}-\theta_{2}-\sigma_{3}\right)+s_{4} \cos \left(2 \theta_{2}-\sigma_{4}\right),
$$

with given non-negative coefficients $s_{i}$, and phases $\sigma_{i}$. If $R$ is a Morse function, i.e. its critical points are all nondegenerate, then the number of such critical points is 8, 12 or 16 .

Proof. In view of the invariance of $R$ under the shift

$$
\left(\theta_{1}, \theta_{2}\right) \mapsto\left(\theta_{1}+\pi, \theta_{2}+\pi\right)
$$

we carry out the following 2-to-1 linear change on $\mathbb{T}^{2}$ :

$$
\psi_{1}=2 \theta_{1}-\sigma_{1}, \quad \psi_{2}=\theta_{1}+\theta_{2}-\sigma_{2} .
$$

Its inverse is a "1-to-2 map":

$$
\theta_{1}=\frac{\psi_{1}+\sigma_{1}}{2}+\nu, \quad \theta_{2}=\psi_{2}-\frac{\psi_{1}+\sigma_{1}}{2}+\sigma_{2}+\nu \quad(\nu=0, \pi)
$$

With this change we obtain

$$
\widetilde{R}\left(\psi_{1}, \psi_{2}\right)=s_{1} \cos \psi_{1}+s_{2} \cos \psi_{2}+s_{3} \cos \left(\psi_{1}-\psi_{2}-\hat{\sigma}_{1}\right)+s_{4} \cos \left(\psi_{1}-2 \psi_{2}-\hat{\sigma}_{2}\right),
$$

with $\hat{\sigma}_{1}=-\sigma_{1}+\sigma_{2}+\sigma_{3}$ and $\hat{\sigma}_{2}=-\sigma_{1}+2 \sigma_{2}-\sigma_{4}$. Since each critical point of $\widetilde{R}$ gives rise to 2 critical points of $R$, we need to prove that $\widetilde{R}$ has 4,6 or 8 critical points.

One can give a lower bound for the number of critical points of $\widetilde{R}$ from some results of Morse theory [11]. Recall that the index of a critical point $p$ is the number of negative eigenvalues for the Hessian matrix for $\widetilde{R}$ at $p$, and denote as $\nu_{k}=\nu_{k}(\widetilde{R})$ the number of critical points of the index $k, 0 \leq k \leq 2$ (then, the amounts $\nu_{0}, \nu_{1}, \nu_{2}$ are the number of minima, saddle points and maxima, respectively). The Morse inequalities connect the number of critical points of a given index and the Betti numbers (ranks of the homology groups of the 
manifold under consideration). For the case of the 2-torus, the Morse inequalities are of the form

$$
\nu_{0} \geq 1, \quad \nu_{1}-\nu_{0} \geq 1, \quad \nu_{2}-\nu_{1}+\nu_{0}=\chi\left(\mathbb{T}^{2}\right)=0,
$$

where $\chi$ is the Euler characteristics. Therefore, the minimal number of critical points of $\widetilde{R}$ (assuming that they are all nondegenerate) is equal to 4 , and for a function with the minimal number of critical points one gets the following distribution of their types: a minimum, two saddles and a maximum. We also deduce that the total number of critical points for a Morse function on $\mathbb{T}^{2}$ is even, since $\nu_{0}+\nu_{1}+\nu_{2}=2 \nu_{1}$.

To find an upper bound for the number of critical points of $\widetilde{R}$, we shall show that the critical points can be reduced to zeroes of a trigonometric polynomial of one angle. We write

$$
\widetilde{R}=\lambda_{0}\left(\psi_{2}\right)+\lambda_{1}\left(\psi_{2}\right) \cos \psi_{1}+\lambda_{2}\left(\psi_{2}\right) \sin \psi_{1},
$$

where we define

$$
\begin{aligned}
& \lambda_{0}\left(\psi_{2}\right)=s_{2} \cos \psi_{2}, \\
& \lambda_{1}\left(\psi_{2}\right)=s_{1}+s_{3} \cos \left(\psi_{2}+\hat{\sigma}_{1}\right)+s_{4} \cos \left(2 \psi_{2}+\hat{\sigma}_{2}\right), \\
& \lambda_{2}\left(\psi_{2}\right)=s_{3} \sin \left(\psi_{2}+\hat{\sigma}_{1}\right)+s_{4} \sin \left(2 \psi_{2}+\hat{\sigma}_{2}\right) .
\end{aligned}
$$

The critical points of $\widetilde{R}$ are the solutions of the following system of equations:

$$
\begin{aligned}
& \frac{\partial \widetilde{R}}{\partial \psi_{1}}=\lambda_{2}\left(\psi_{2}\right) \cos \psi_{1}-\lambda_{1}\left(\psi_{2}\right) \sin \psi_{1}=0, \\
& \frac{\partial \widetilde{R}}{\partial \psi_{2}}=\lambda_{1}^{\prime}\left(\psi_{2}\right) \cos \psi_{1}+\lambda_{2}^{\prime}\left(\psi_{2}\right) \sin \psi_{1}+\lambda_{0}^{\prime}\left(\psi_{2}\right)=0 .
\end{aligned}
$$

This can be interpreted as a linear system for $\cos \psi_{1}$, $\sin \psi_{1}$, whose determinant is

$$
\Delta\left(\psi_{2}\right)=\lambda_{1} \lambda_{1}^{\prime}+\lambda_{2} \lambda_{2}^{\prime}=\frac{1}{2}\left(\lambda_{1}^{2}+\lambda_{2}^{2}\right)^{\prime}
$$

If a critical point $\left(\psi_{1}, \psi_{2}\right)$ satisfies $\Delta\left(\psi_{2}\right) \neq 0$, solving the linear system $(20-21)$ we get

$$
\cos \psi_{1}=-\frac{\lambda_{0}^{\prime} \lambda_{1}}{\lambda_{1} \lambda_{1}^{\prime}+\lambda_{2} \lambda_{2}^{\prime}}, \quad \sin \psi_{1}=-\frac{\lambda_{0}^{\prime} \lambda_{2}}{\lambda_{1} \lambda_{1}^{\prime}+\lambda_{2} \lambda_{2}^{\prime}},
$$

and the equality $\cos ^{2} \psi_{1}+\sin ^{2} \psi_{1}=1$ implies that $\psi_{2}$ must be a zero of the following trigonometric polynomial:

$$
g\left(\psi_{2}\right)=\left(\lambda_{1} \lambda_{1}^{\prime}+\lambda_{2} \lambda_{2}^{\prime}\right)^{2}-\left(\lambda_{0}^{\prime}\right)^{2}\left(\lambda_{1}^{2}+\lambda_{2}^{2}\right)=0 .
$$

One can easily check that $\lambda_{1}^{2}+\lambda_{2}^{2}$ is a trigonometric polynomial in $\psi_{2}$ of degree $\leq 2$ (indeed, one can use (18-19) and check that all the terms of degrees 3 and 4 cancel). Taking a derivative it is clear that the same is true for $\lambda_{1} \lambda_{1}^{\prime}+\lambda_{2} \lambda_{2}^{\prime}$. Then, we see from (23) that $g\left(\psi_{2}\right)$ is a trigonometric polynomial of degree $\leq 4$, which gives at most 8 possible values for $\psi_{2}$ 
(unless $g\left(\psi_{2}\right)$ is identically zero; see below). Replacing each value of $\psi_{2}$ into (22), one single value of $\psi_{1}$ is determined. This implies that the function $\widetilde{R}$ has at most 8 critical points with $\Delta\left(\psi_{2}\right) \neq 0$.

Of course, additional critical points $\left(\psi_{1}, \psi_{2}\right)$ with $\Delta\left(\psi_{2}\right)=0$ may occur. But in this case we must have in (20-21) that $\lambda_{1}\left(\psi_{2}\right)=\lambda_{2}\left(\psi_{2}\right)=0$ or $\lambda_{0}^{\prime}\left(\psi_{2}\right)=0$. In both subcases, we see that (23) is also satisfied, now with $\psi_{2}$ as a double zero. Replacing $\psi_{2}$ into (20-21), we obtain at most two values for $\psi_{1}$ (unless $\lambda_{1}, \lambda_{1}^{\prime}, \lambda_{2}, \lambda_{2}^{\prime}$ simultaneously vanish at $\psi_{2}$, but in this case one has a line of critical points). Then, even if $\Delta\left(\psi_{2}\right)=0$ for some of the critical points, we deduce that the total number of critical points for $\widetilde{R}$ cannot be larger than 8 .

In the preceding discussion, one should consider the possibility that $g\left(\psi_{2}\right)$ be identically zero. We are going to show that, if this happens, then there appears a curve formed with critical points. Assuming $g\left(\psi_{2}\right) \equiv 0$, if $\Delta\left(\psi_{2}\right) \neq 0$ in some interval, we deduce from (22) that for each $\psi_{2}$ in this interval one value of $\psi_{1}$ is determined, obtaining a curve of critical points. Otherwise, if $g\left(\psi_{2}\right) \equiv 0$ and $\Delta\left(\psi_{2}\right) \equiv 0$, we deduce that $\lambda_{1}\left(\psi_{2}\right)=\lambda_{2}\left(\psi_{2}\right) \equiv 0$ in some interval, or $\lambda_{0}^{\prime}\left(\psi_{2}\right) \equiv 0$ in some interval. In the first case we see from (18-19) that $s_{1}=s_{3}=s_{4}=0$, and clearly there are lines of critical points. In the second case, we obtain from (20-21) that for each $\psi_{2}$ two values of $\psi_{1}$ are determined, giving rise to two curves of critical points. Thus, if $g\left(\psi_{2}\right) \equiv 0$ there are always curves of critical points, and this contradicts the assumption that $\widetilde{R}$ is a Morse function (for such a function, all critical points must be isolated).

We deduce from these arguments that the number of critical points of $\widetilde{R}$ must be 4,6 or 8. After the linear change (16), the number of critical points of $R$ is doubled: 8,12 or 16 .

In an analogous way to Theorem 1 , one can study the case when, for any $c$,

$$
\alpha(0, c)=\left.\frac{d}{d \varepsilon}\right|_{\varepsilon=0}(\varepsilon \alpha(\varepsilon, c)) \equiv 0^{2}
$$

(see (10)). This case is reduced to the preceding one, as terms containing $\alpha_{i}(0, c)$ vanish and, after dividing by $\sqrt{I_{1}^{0} I_{2}^{0}}$, we get the same system (13).

Now consider a generic one-parameter unfolding. We will show here that the perturbed system has four transverse (on the related level of the Hamiltonian) homoclinic orbits to every perturbed persistent Diophantine tori $I_{1}=I_{1}^{0}, I_{2}=I_{2}^{0}$ on the center manifold $W^{c}$, if $\left(I_{1}^{0}, I_{2}^{0}\right)$ belong to some set in the plane $\left(I_{1}, I_{2}\right)$.

Theorem 2 There are $\varepsilon_{0}>0$ and some open set $D$ in the quadrant $I_{1}>0, I_{2}>0$ such that for $\left(I_{1}^{0}, I_{2}^{0}\right) \in D$ a persistent Diophantine torus $I_{1}=I_{1}^{0}, I_{2}=I_{2}^{0}$ has exactly 4 transverse (in the related level of Hamiltonian) homoclinic orbits to this torus.

Proof. To find homoclinic orbits to a persistent invariant torus, given by $I_{1}=I_{1}^{0}, I_{2}=I_{2}^{0}$, we need to find intersections between its stable and unstable manifolds or equivalently, as in Theorem 1, solutions of system (12).

\footnotetext{
${ }^{2}$ The sense of this identity is that one considers an one-parameter unfolding of $H_{0}$ such that the related path in the space of all Hamiltonians is tangent at the point $H_{0}$ to $\mathcal{L}$.
} 
Dividing the first equation of system (12) by $\sqrt{2 I_{1}^{0}}$, and the second one by $\sqrt{2 I_{2}^{0}}$, the system casts into the form

$$
\begin{aligned}
& \alpha_{1}(0,0) \cos \theta_{1}+\alpha_{3}(0,0) \sin \theta_{1}+O\left(\left\|I^{0}\right\|^{1 / 2}\right)=0, \\
& \alpha_{2}(0,0) \cos \theta_{2}+\alpha_{4}(0,0) \sin \theta_{2}+O\left(\left\|I^{0}\right\|^{1 / 2}\right)=0 .
\end{aligned}
$$

Tending in (24) to the limit $I_{1}^{0} \rightarrow 0, I_{2}^{0} \rightarrow 0$, we obtain the system

$$
\begin{gathered}
\alpha_{1}(0,0) \cos \theta_{1}+\alpha_{3}(0,0) \sin \theta_{1}=0, \\
\alpha_{2}(0,0) \cos \theta_{2}+\alpha_{4}(0,0) \sin \theta_{2}=0
\end{gathered}
$$

(notice that, as in the proof of Theorem 1, this system can be put in gradient form). We assume (it is the condition of the general position) that

$$
\alpha_{1}(0,0)^{2}+\alpha_{3}(0,0)^{2} \neq 0, \quad \alpha_{2}(0,0)^{2}+\alpha_{4}(0,0)^{2} \neq 0 .
$$

Then, the system has four solutions $p^{k}=\left(\theta_{1}^{k}, \theta_{2}^{k}\right), k=\overline{1,4}$ (we stress that, in this case, the solutions do not depend on the ray to which $\left(I_{1}^{0}, I_{2}^{0}\right)$ belongs). Due to (LLL) the implicit function theorem is applicable to (24), and gives four solutions $p^{k}=\left(\theta_{1}^{k}\left(I_{1}^{0}, I_{2}^{0}\right), \theta_{2}^{k}\left(I_{1}^{0}, I_{2}^{0}\right)\right)$, $k=\overline{1,4}$. In fact, only a positive measure Cantorian set of tori persists, but the implicit function theorem works here uniformly in $I$ out of some neighborhood of the former homoclinic orbit.

\section{The Melnikov potential}

The method of detecting homoclinic orbits to invariant tori presented in previous section is, in a sense, blind: it is rather hard to apply it to concrete Hamiltonians and perturbations. Now, we are interested in detecting the effective existence of transverse homoclinic orbits for concrete perturbations $\varepsilon H_{1}$, as well as in obtaining a first approximation for (the traces in $N^{s}$ of) these orbits. The standard procedure for this purpose is the Poincaré-Melnikov method, which provides the Melnikov function as an approximation to the splitting distance (i.e. the distance between the stable and unstable manifolds of a given torus).

The transverse homoclinic orbits are usually approximated as simple zeroes of the (vector) Melnikov function but, as in [4], the Hamiltonian character of the equations allows us to look for nondegenerate critical points of the (scalar) Melnikov potential, whose gradient is the Melnikov function.

For the sake of simplicity, we restrict ourselves to the case of an unperturbed Hamiltonian $H_{0}$ as in the examples considered in Section 1.1, plus a perturbation $\varepsilon H_{1}\left(x_{1}, y_{1}, x_{2}, y_{2}, p, q\right)$.

We know from Section 3 that the persistent tori and their invariant manifolds become transparent in the local KAM coordinates introduced there. However, since the Melnikov function will be defined with the help of integrals along trajectories on the global unperturbed homoclinic manifold, it is natural to use the global original coordinates $z=\left(x_{1}, y_{1}, x_{2}, y_{2}, p, q\right)$ in order to deduce an expression for the Melnikov function. To be more precise, we are 
going to use the coordinates $\zeta=\left(\xi_{1}, \xi_{2}, \varphi_{1}, \varphi_{2}, p, q\right)$, i.e. we change to the polar coordinates introduced in (5).

The reason for using polar coordinates is that the Melnikov function will provide a first approximation for the splitting distance along the actions $\xi=\left(\xi_{1}, \xi_{2}\right)$, which are first integrals of $H_{0}$. Notice that the unperturbed homoclinic manifold of a torus is given by $\{\xi=$ const, $p=$ $0\}$, and it will be enough to give a measure for the splitting along the $\xi$-directions due to the conservation of energy.

First, we describe the parameterizations to be used. We denote $\mathcal{T}$ a persistent Diophantine torus in $W^{c}$, with actions $I^{0}=\left(I_{1}^{0}, I_{2}^{0}\right)$. This torus can be parameterized by $\theta=\left(\theta_{1}, \theta_{2}\right) \in$ $\mathbb{T}^{2}$ in the coordinates provided by KAM theorem in Section 3. Let $\mathcal{Z}^{*}\left(I^{0}, \theta, \varepsilon\right)$ be the parameterization of the torus $\mathcal{T}$ in the coordinates $\zeta$, and let $\mathcal{I}^{*}\left(I^{0}, \theta, \varepsilon\right)=\left(\mathcal{I}_{1}^{*}\left(I^{0}, \theta, \varepsilon\right), \mathcal{I}_{2}^{*}\left(I^{0}, \theta, \varepsilon\right)\right)$ denote its $\xi$-components: $\mathcal{Z}^{*}=\left(\mathcal{I}_{1}^{*}, \mathcal{I}_{2}^{*}, \ldots\right)$.

Let us denote $\mathcal{A}^{s}, \mathcal{A}^{u}$ the traces of the torus $\mathcal{T}$ on the transverse sections $N^{s}, N^{u}$ (i.e. the intersection of the stable and unstable manifolds of $\mathcal{T}$ with $N^{s}, N^{u}$ ). Notice that these traces are 2-dimensional tori. Recall that the transverse sections have been defined in (2) by a number $d$, not depending on $\varepsilon$. This $d$ can be chosen small enough in such a way that the traces are contained in the domain of validity of the KAM results of Section 3. We use for the traces $\mathcal{A}^{s, u}$ the same parameters as for the torus $\mathcal{T}$ : as before, we denote $\mathcal{Z}^{s, u}\left(I^{0}, \theta, \varepsilon\right)$ the whole parameterization, and $\mathcal{I}^{s, u}=\left(\mathcal{I}_{1}^{s, u}, \mathcal{I}_{2}^{s, u}\right)$ their $\xi$-components. We see from KAM Hamiltonian equations (9) that the angles $\theta \in \mathbb{T}^{2}$ in the parameterizations $\mathcal{Z}^{*}$, $\mathcal{Z}^{s}, \mathcal{Z}^{u}$ can be taken in such a way that the trajectory starting at the point $\mathcal{Z}^{s}\left(I^{0}, \theta, \varepsilon\right)$ is asymptotic for $t \rightarrow \infty$ to the trajectory starting at $\mathcal{Z}^{*}\left(I^{0}, \theta, \varepsilon\right)$, and the trajectory starting at $\mathcal{Z}^{u}\left(I^{0}, \theta, \varepsilon\right)$ is asymptotic for $t \rightarrow-\infty$ to the trajectory starting at $\mathcal{Z}^{*}\left(I^{0}, \theta, \varepsilon\right)$.

Now we consider the image of $\mathcal{A}^{u}$ through the global map $S_{\varepsilon}: N^{u} \rightarrow N^{s}$, and denote $\overline{\mathcal{Z}}^{u}\left(I^{0}, \theta, \varepsilon\right)$ the parameterization of $S_{\varepsilon}\left(\mathcal{A}^{u}\right)$ inherited from $\mathcal{A}^{u}$, i.e. $\overline{\mathcal{Z}}^{u}$ is the point where the trajectory starting at $\mathcal{Z}^{u}$ intersects $N^{s}$. As before, we denote $\overline{\mathcal{I}}^{u}=\left(\overline{\mathcal{I}}_{1}^{u}, \overline{\mathcal{I}}_{2}^{u}\right)$ the $\xi$ components of the point $\overline{\mathcal{Z}}^{u}$. As a measure for the splitting distance, we are going to take the distance between the tori $\mathcal{A}^{s}$ and $S_{\varepsilon}\left(\mathcal{A}^{u}\right)$, restricted to the $\xi_{i}$-directions. Nevertheless, the phase drift that occurs along any trajectory has to be taken into account in order to measure this distance correctly.

Since the trajectories on the invariant manifolds of the torus $\mathcal{T}$ are close to the unperturbed homoclinic trajectories, we look at the case $\varepsilon=0$ in order to choose the phases. Recalling the function $q_{0}(t)$ in the examples given in Section 1.1, a homoclinic trajectory to $\mathcal{T}$ for $H_{0}$ is given by

$$
\zeta_{0}(t)=\zeta_{0}\left(t, I^{0}, \theta\right)=\left(I^{0}, \theta+t \tilde{\omega}, \dot{q}_{0}(t), q_{0}(t)\right)
$$

where we have denoted $\tilde{\omega}=\left(\tilde{\omega}_{1}, \tilde{\omega}_{2}\right)$, with

$$
\tilde{\omega}_{1}=\frac{\partial h_{0}}{\partial \xi_{1}}\left(I_{1}^{0}, I_{2}^{0}\right)=\omega_{1}+2 A I_{1}^{0}+2 B I_{2}^{0}+\cdots, \quad \tilde{\omega}_{2}=\frac{\partial h_{0}}{\partial \xi_{2}}\left(I_{1}^{0}, I_{2}^{0}\right)=\omega_{2}+2 B I_{1}^{0}+2 C I_{2}^{0}+\cdots
$$

It is clear that trajectory (25) is asymptotic for $t \rightarrow \pm \infty$, with exponential estimates, to the following trajectory on the unperturbed torus:

$$
\zeta_{0}^{*}(t)=\zeta_{0}^{*}\left(t, I^{0}, \theta\right)=\left(I^{0}, \theta+t \tilde{\omega}, 0,0\right) .
$$


Let $T_{1}, T_{2}>0$ constants such that $\zeta_{0}\left(-T_{1}\right) \in N^{u}, \zeta_{0}\left(T_{2}\right) \in N^{s}$. We have $T_{1}, T_{2} \sim \ln (1 / d)$, where $d$ is the number (chosen small enough) defining the sections $N^{s}, N^{u}$.

Remark 3 It is worth stressing the fact that at $\varepsilon=0$, the Hamiltonian $H_{0}$ in the examples considered is the sum of two independent subHamiltonians $h_{0}$ and $p^{2} / 2+V(q)$ (decoupled subsystems). In this case all homoclinic orbits to a fixed torus in $W^{c}$ possess the property of the same asymptotics (the same orbit on the torus) as $t \rightarrow+\infty$ and $t \rightarrow-\infty$. For the case of coupled integrable Hamiltonians these asymptotic orbits are different generically, and this has to be taken into account for the Melnikov potential, which becomes the sum of two distinct integrals for $t<0$ and $t>0$. In fact, the Poincaré-Melnikov method for more general unperturbed Hamiltonians $H_{0}$ was developed in [4] introducing an alternative single integral expression for the Melnikov potential (a previous work in this direction was [15]).

Now, we can define the following (vector) function as an exact measure for the splitting distance:

$$
\mathcal{M}\left(I^{0}, \theta, \varepsilon\right)=\left(\overline{\mathcal{I}}^{u}\left(I^{0}, \theta-T_{1} \tilde{\omega}, \varepsilon\right)-\mathcal{I}^{s}\left(I^{0}, \theta+T_{2} \tilde{\omega}, \varepsilon\right)\right) .
$$

The next theorem provides a first order approximation for the function $\mathcal{M}$ in terms of the gradient of a scalar function, called the Melnikov potential, defined as follows:

$$
L\left(I^{0}, \theta\right)=-\int_{-\infty}^{\infty}\left[\hat{H}_{1}\left(\zeta_{0}(t)\right)-\hat{H}_{1}\left(\zeta_{0}^{*}(t)\right)\right] d t
$$

where $\hat{H}_{1}(\zeta)$ denotes the perturbation $H_{1}$ expressed in the coordinates $\zeta$. The integral is absolutely convergent since $\zeta_{0}(t)$ is asymptotic for $t \rightarrow \pm \infty$ to $\zeta_{0}^{*}(t)$ (with exponential estimates).

Theorem 3 For the splitting distance along the $\xi_{i}$-directions, the following first order approximation holds:

$$
\mathcal{M}=\varepsilon \nabla_{\theta} L+\mathcal{O}\left(\varepsilon^{2}\right)
$$

Proof. For any $\theta=\left(\theta_{1}, \theta_{2}\right)$, let us consider the trajectories $\zeta^{s, u}(t)=\zeta^{s, u}\left(t, I^{0}, \theta, \varepsilon\right)$ such that $\zeta^{u}\left(-T_{1}\right)=\mathcal{Z}^{u}\left(I^{0}, \theta-T_{1} \tilde{\omega}, \varepsilon\right)$ and $\zeta^{s}\left(T_{2}\right)=\mathcal{Z}^{s}\left(I^{0}, \theta+T_{2} \tilde{\omega}, \varepsilon\right)$. The trajectories $\zeta^{s}(t)$ and $\zeta^{u}(t)$ are $\mathcal{O}(\varepsilon)$-close to $\zeta_{0}(t)$ for $t \geq T_{2}$ and $t \leq-T_{1}$ respectively, due to that these parts of the trajectories are contained in the domain of validity of the KAM results, and they are asymptotic to the torus as $t \rightarrow \pm \infty$. Extending these estimates to $-T_{1} \leq t \leq T_{2}$ (an interval whose length is $\sim \ln (1 / d)$, outside of the scope of KAM results), we see from Gronwall inequality that the trajectory $\zeta^{u}(t)$ is $\mathcal{O}\left(\varepsilon / d^{\alpha}\right)$-close to $\zeta_{0}(t)$ for some $\alpha>0 .^{3}$ However, since the number $d$ does not depend on $\varepsilon$, it follows that $\zeta^{u}(t)$ is $\mathcal{O}(\varepsilon)$-close to $\zeta_{0}(t)$ for all $t \leq T_{2}$. Using this estimate, and the fact that the trajectory $\zeta^{u}(t)$ intersects $N^{s}$ transversely, we deduce that $\overline{\mathcal{Z}}^{u}=\zeta^{u}\left(T_{2}+\mathcal{O}(\varepsilon)\right)$ and, for the actions, $\overline{\mathcal{I}}_{i}^{u}=\xi_{i}^{u}\left(T_{2}\right)+\mathcal{O}\left(\varepsilon^{2}\right)$ since $\dot{\xi}_{i}=\mathcal{O}(\varepsilon)$.

\footnotetext{
${ }^{3}$ This $\alpha$ is given by the Lipschitz constant for the Hamiltonian vector field associated to $H_{0}$; it can be obtained from a bound (on a compact set) for the second derivatives of $H_{0}$.
} 
On the torus $\mathcal{T}$, let $\zeta^{*}(t)=\zeta^{*}\left(t, I^{0}, \theta, \varepsilon\right)$ denote the trajectory to which $\zeta^{s}(t)$ is asymptotic for $t \rightarrow \infty$, with $\zeta^{*}\left(T_{2}\right)=\mathcal{Z}^{*}\left(I^{0}, \theta+T_{2} \tilde{\omega}, \varepsilon\right)$. This trajectory $\zeta^{*}(t)$ is $\mathcal{O}(\varepsilon)$-close to $\zeta_{0}^{*}(t)$ for all $t \in \mathbb{R}$. The trajectory $\zeta^{u}(t)$ is also asymptotic for $t \rightarrow-\infty$ to the trajectory $\zeta^{*}(t)$.

For the components of the function $\mathcal{M}$, we write

$$
\overline{\mathcal{I}}_{i}^{u}-\mathcal{I}_{i}^{s}=\left(\overline{\mathcal{I}}_{i}^{u}-\mathcal{I}_{i}^{*}\right)-\left(\mathcal{I}_{i}^{s}-\mathcal{I}_{i}^{*}\right)=\left(\xi_{i}^{u}\left(T_{2}\right)-\xi_{i}^{*}\left(T_{2}\right)\right)-\left(\xi_{i}^{s}\left(T_{2}\right)-\xi_{i}^{*}\left(T_{2}\right)\right)+\mathcal{O}\left(\varepsilon^{2}\right) .
$$

To obtain the first order approximation, we use that $\xi_{1}, \xi_{2}$ are first integrals of $H_{0}$ in a neighborhood $U$ of the singular point $O$ :

$$
\dot{\xi}_{i}=\varepsilon\left\{\xi_{i}, \hat{H}_{1}\right\}=-\varepsilon \frac{\partial \hat{H}_{1}}{\partial \varphi_{i}}=\varepsilon\left(y_{i} \frac{\partial H_{1}}{\partial x_{i}}-x_{i} \frac{\partial H_{1}}{\partial y_{i}}\right) .
$$

For the "stable" term in (28), we obtain:

$$
\begin{gathered}
-\left(\xi_{i}^{s}\left(T_{2}\right)-\xi_{i}^{*}\left(T_{2}\right)\right)=\int_{T_{2}}^{\infty}\left[\dot{\xi}_{i}^{s}(t)-\dot{\xi}_{i}^{*}(t)\right] d t=-\varepsilon \int_{T_{2}}^{\infty}\left[\frac{\partial \hat{H}_{1}}{\partial \varphi_{i}}\left(\zeta^{s}(t)\right)-\frac{\partial \hat{H}_{1}}{\partial \varphi_{i}}\left(\zeta^{*}(t)\right)\right] d t \\
=-\varepsilon \int_{T_{2}}^{\infty}\left[\frac{\partial \hat{H}_{1}}{\partial \varphi_{i}}\left(\zeta_{0}(t)\right)-\frac{\partial \hat{H}_{1}}{\partial \varphi_{i}}\left(\zeta_{0}^{*}(t)\right)\right] d t+\mathcal{O}\left(\varepsilon^{2}\right),
\end{gathered}
$$

where we have used the $\mathcal{O}(\varepsilon)$-closeness of the perturbed trajectories to the unperturbed ones, for $t \geq T_{2}$. The integral involved is absolutely convergent, since $\zeta^{s}(t)$ is asymptotic for $t \rightarrow \infty$ to $\zeta^{*}(t)$ with exponential estimates. For the "unstable" term in (28), we can proceed analogously (although we integrate in a "longer" interval, this does not make the estimates worse, because the length of the additional interval $-T_{1} \leq t \leq T_{2}$ does not depend on $\varepsilon$ ), and we obtain:

$$
\begin{gathered}
\xi_{i}^{u}\left(T_{2}\right)-\xi_{i}^{*}\left(T_{2}\right)=\int_{-\infty}^{T_{2}}\left[\dot{\xi}_{i}^{u}(t)-\dot{\xi}_{i}^{*}(t)\right] d t=-\varepsilon \int_{-\infty}^{T_{2}}\left[\frac{\partial \hat{H}_{1}}{\partial \varphi_{i}}\left(\zeta^{u}(t)\right)-\frac{\partial \hat{H}_{1}}{\partial \varphi_{i}}\left(\zeta^{*}(t)\right)\right] d t \\
=-\varepsilon \int_{-\infty}^{T_{2}}\left[\frac{\partial \hat{H}_{1}}{\partial \varphi_{i}}\left(\zeta_{0}(t)\right)-\frac{\partial \hat{H}_{1}}{\partial \varphi_{i}}\left(\zeta_{0}^{*}(t)\right)\right] d t+\mathcal{O}\left(\varepsilon^{2}\right) .
\end{gathered}
$$

Replacing in (28) the approximations obtained, we obtain an integral $\int_{-\infty}^{\infty}$ (the Melnikov function) that gives a first order approximation to the function $\mathcal{M}$, with an error term $\mathcal{O}\left(\varepsilon^{2}\right)$. This integral is the gradient in $\left(\theta_{1}, \theta_{2}\right)$ of the Melnikov potential defined in (26).

Since the splitting distance is approximated by the gradient of the Melnikov potential $L$, the nondegenerate critical points in $\theta=\left(\theta_{1}, \theta_{2}\right)$ of $L$ will give rise to transverse homoclinic orbits. We point out that, in related cases, it can be shown [4] that the whole splitting distance (and not only its first order approximation) is also the gradient of a scalar function, called the splitting potential (due to the Hamiltonian character of the equations, which implies Lagrangian properties for the invariant manifolds). This fact is very useful in order to ensure the existence of homoclinic orbits in degenerate (non-transverse) cases, but we do not need it here. 
Now, we are going to study the nondegenerate critical points in $\theta$ of the Melnikov potential. This requires explicit computations and, to give an illustration, we only consider the case of the pendulum (example 1, among the three ones considered in Section 1.1), and a more concrete perturbation:

$$
H_{1}=(\cos q-\nu) f\left(x_{1}, y_{1}, x_{2}, y_{2}\right)
$$

where $\nu$ is a parameter. It is not hard to see that for $\nu=1$ all tori persist with their local invariant manifolds, whereas for $\nu \neq 1$ we have a majority of persistent Diophantine tori from KAM theory. In both cases, the global invariant manifolds may not coincide, and to study their transverse intersections we use the Melnikov potential. Applying (26) and using that

$$
\cos q_{0}(t)-1=-\frac{2}{\cosh ^{2} t}
$$

we obtain the following expression for the Melnikov potential, which does not depend on the value of $\nu$ :

$$
L\left(I^{0}, \theta\right)=2 \int_{-\infty}^{\infty} \frac{\hat{f}\left(I^{0}, \theta+t \tilde{\omega}\right)}{\cosh ^{2} t} d t .
$$

In the next two theorems, two different types of functions $f$ are considered in the perturbation (30), and the existence and number of transverse homoclinic orbits for such a perturbation is studied. First, in Theorem 4 , we consider $f=\mathcal{O}_{2}\left(x_{1}, y_{1}, x_{2}, y_{2}\right)$, and writing down the Hamiltonian equations one easily checks that the homoclinic loop $\Gamma$ persists (to be more precise, it is kept unchanged). Instead, in Theorem 5 we consider a more general function $f$ and, in general, the loop will not be preserved. Their statements are similar to those of Theorems 1 and 2, but now we provide a condition that can be checked for concrete examples.

We recall that a scalar function on a manifold is called a Morse function provided all its critical points are nondegenerate.

Theorem 4 Let $f=\mathcal{O}_{2}\left(x_{1}, y_{1}, x_{2}, y_{2}\right)$ in the perturbation (30), denote $f_{2}$ its quadratic part (i.e. $\left.f=f_{2}+\mathcal{O}_{3}\right)$. For any action $I^{0}=\left(I_{1}^{0}, I_{2}^{0}\right)$, let us denote $L_{2}\left(I^{0}, \cdot\right)$ the part of the Melnikov potential coming from $f_{2}$, and let $\mathcal{B}$ be the set of actions $I^{0}$ such that $L_{2}\left(I^{0}, \cdot\right)$ is a Morse function of $\theta \in \mathbb{T}^{2}$. Then, for $I^{0}$ in an open subset of $\mathcal{B}$, and $\varepsilon$ small enough, there exist either 8, 12 or 16 transverse homoclinic orbits.

Proof. Let $f=b_{1} x_{1}^{2}+b_{2} x_{1} y_{1}+b_{3} x_{1} x_{2}+b_{4} x_{1} y_{2}+b_{5} y_{1}^{2}+b_{6} y_{1} x_{2}+b_{7} y_{1} y_{2}+b_{8} x_{2}^{2}+b_{9} x_{2} y_{2}+$ $b_{10} y_{2}^{2}+\mathcal{O}_{3}\left(x_{1}, y_{1}, x_{2}, y_{2}\right)$. In polar coordinates, this function becomes

$$
\begin{aligned}
\hat{f}\left(\xi_{1}, \xi_{2}, \varphi_{1}, \varphi_{2}\right) & \\
= & \xi_{1}\left[\left(b_{1}+b_{5}\right)+\left(b_{1}-b_{5}\right) \cos 2 \varphi_{1}+b_{2} \sin 2 \varphi_{1}\right] \\
& +\sqrt{\xi_{1} \xi_{2}}\left[\left(b_{3}-b_{7}\right) \cos \left(\varphi_{1}+\varphi_{2}\right)+\left(b_{4}+b_{6}\right) \sin \left(\varphi_{1}+\varphi_{2}\right)\right. \\
& \left.\quad+\left(b_{3}+b_{7}\right) \cos \left(\varphi_{1}-\varphi_{2}\right)-\left(b_{4}-b_{6}\right) \sin \left(\varphi_{1}-\varphi_{2}\right)\right] \\
& +\xi_{2}\left[\left(b_{8}+b_{10}\right)+\left(b_{8}-b_{10}\right) \cos 2 \varphi_{2}+b_{9} \sin 2 \varphi_{2}\right]+\mathcal{O}\left(\|\xi\|^{3 / 2}\right) .
\end{aligned}
$$


Inserting this expansion into the integral and taking into account that integrals of odd functions vanish, and using also that $\tilde{\omega}_{i}=\omega_{i}+\mathcal{O}\left(\left\|I^{0}\right\|\right)$, we get

$$
\begin{aligned}
L\left(I_{1}^{0}, I_{2}^{0}, \theta_{1}, \theta_{2}\right) & \\
=\quad & 2 I_{1}^{0}\left[2\left(b_{1}+b_{5}\right)+\mathcal{J}_{2 \omega_{1}}\left(\left(b_{1}-b_{5}\right) \cos 2 \theta_{1}+b_{2} \sin 2 \theta_{1}\right)\right] \\
& +2 \sqrt{I_{1}^{0} I_{2}^{0}}\left[\mathcal{J}_{\omega_{1}+\omega_{2}}\left(\left(b_{3}-b_{7}\right) \cos \left(\theta_{1}+\theta_{2}\right)+\left(b_{4}+b_{6}\right) \sin \left(\theta_{1}+\theta_{2}\right)\right)\right. \\
& \left.\quad+\mathcal{J}_{\omega_{1}-\omega_{2}}\left(\left(b_{3}+b_{7}\right) \cos \left(\theta_{1}-\theta_{2}\right)-\left(b_{4}-b_{6}\right) \sin \left(\theta_{1}-\theta_{2}\right)\right)\right] \\
& +2 I_{2}^{0}\left[2\left(b_{8}+b_{10}\right)+\mathcal{J}_{2 \omega_{2}}\left(\left(b_{8}-b_{10}\right) \cos 2 \theta_{2}+b_{9} \sin 2 \theta_{2}\right)\right]+\mathcal{O}\left(\left\|I^{0}\right\|^{3 / 2}\right),
\end{aligned}
$$

where we define

$$
\mathcal{J}_{a}=\int_{-\infty}^{\infty} \frac{\cos a t}{\cosh ^{2} t} d t=\frac{\pi a}{\sinh (\pi a / 2)}, \quad a \neq 0,
$$

computed by residue theory. We have also used that $\mathcal{J}_{0}=2$. The Melnikov potential (32) becomes a generic function of the type

$$
\begin{aligned}
L=L_{2} & +\mathcal{O}\left(\left\|I^{0}\right\|^{3 / 2}\right), \\
L_{2}=s_{0} & +s_{1} \cos \left(2 \theta_{1}-\sigma_{1}\right)+s_{2} \cos \left(\theta_{1}+\theta_{2}-\sigma_{2}\right) \\
& +s_{3} \cos \left(\theta_{1}-\theta_{2}-\sigma_{3}\right)+s_{4} \cos \left(2 \theta_{2}-\sigma_{4}\right),
\end{aligned}
$$

where the (non-negative) coefficients $s_{i}$ are homogeneous linear polynomials in $I_{1}^{0}, \sqrt{I_{1}^{0} I_{2}^{0}}$, $I_{2}^{0}$, and the phases $\sigma_{i}$ do not depend on $I^{0}$. Since we are interested in the critical points of $L$ with respect to $\theta_{1}, \theta_{2}$, we can obviously assume $s_{0}=0$.

By Lemma 2, if $L_{2}$ is a Morse function it has 8,12 or 16 critical points. This result can be extended to the whole Melnikov potential $L$ in (34) provided $\left\|I^{0}\right\|$ is small enough, excluding a neighborhood of the rays $I_{1}^{0} / I_{2}^{0}=$ const where the Morse condition is not fulfilled. As in Theorem 1, the critical points tend to a constant along each ray, as $\left(I_{1}^{0}, I_{2}^{0}\right) \rightarrow(0,0)$.

Although this theorem does not give explicit conditions determining the number of homoclinic orbits, it is possible to study this in concrete examples. As an illustration, we consider the perturbation (30) with the function

$$
f=\frac{1}{8 \mathcal{J}_{2 \omega_{1}}}\left(x_{1}^{2}-y_{1}^{2}\right)+\frac{\beta}{4 \mathcal{J}_{\omega_{1}+\omega_{2}}}\left(x_{1} x_{2}-y_{1} y_{2}\right)+\frac{\beta}{4 \mathcal{J}_{\omega_{1}-\omega_{2}}}\left(x_{1} x_{2}+y_{1} y_{2}\right)+\frac{1}{8 \mathcal{J}_{2 \omega_{2}}}\left(x_{2}^{2}-y_{2}^{2}\right),
$$

with $\omega_{1}, \omega_{2}$ fixed, and $\beta>0$ as a parameter (recall that $\mathcal{J}_{a}$ has been defined in (33)). The Melnikov potential in (32) becomes

$$
L=L_{2}=\sqrt{I_{1}^{0} I_{2}^{0}}\left(\frac{r}{2} \cos 2 \theta_{1}+\beta \cos \left(\theta_{1}+\theta_{2}\right)+\beta \cos \left(\theta_{1}-\theta_{2}\right)+\frac{1}{2 r} \cos 2 \theta_{2}\right),
$$

with $r=\sqrt{I_{1}^{0} / I_{2}^{0}}$. Removing the term $\sqrt{I_{1}^{0} I_{2}^{0}}$ (that does not influence the critical points of $L$, unless it vanishes), the coefficients considered in the proof of Lemma 2 become $s_{1}=r / 2$, 
$s_{2}=s_{3}=\beta, s_{4}=1 / 2 r$, and the phases are all $\sigma_{i}=0$. After the linear change (16), we obtain $\widetilde{L}_{2}\left(\psi_{1}, \psi_{2}\right)$ as in (17), with

$$
\begin{aligned}
& \lambda_{0}\left(\psi_{2}\right)=\beta \cos \psi_{2}, \\
& \lambda_{1}\left(\psi_{2}\right)=\frac{r}{2}+\beta \cos \psi_{2}+\frac{1}{2 r} \cos 2 \psi_{2}, \\
& \lambda_{2}\left(\psi_{2}\right)=\beta \sin \psi_{2}+\frac{1}{2 r} \sin 2 \psi_{2} .
\end{aligned}
$$

After some computations, we see that the trigonometric polynomial in (23) can be written as

$$
g\left(\psi_{2}\right)=\left(1-\beta^{2}\right) \sin ^{2} \psi_{2}\left(\cos ^{2} \psi_{2}+\beta\left(r+\frac{1}{r}\right) \cos \psi_{2}+\beta^{2}\right) .
$$

It is clear that $g\left(\psi_{2}\right)$ has $\psi_{2}=0, \pi$ as double zeroes, which give rise from (20-21) to 4 critical points: $(0,0),(0, \pi),(\pi, 0),(\pi, \pi)$, for the function $\widetilde{L}_{2}$. Besides, there can exist additional critical points for this function, associated to zeroes $\psi_{2}$ given by $\cos \psi_{2}=-\beta / r$ and $\cos \psi_{2}=$ $-\beta r$. The number of such zeroes can be 0,2 or 4 (excluding degenerate cases), and we deduce that the Melnikov potential (35) may have 8, 12 or 16 critical points. More precisely, we have:

- For $0<\beta<1$, there are 16 critical points if $\beta<r<1 / \beta, r \neq 1$, and 12 critical points if $r<\beta$ or $r>1 / \beta$.

- For $\beta>1$, there are 12 critical points if $r<1 / \beta$ or $r>\beta$, and 8 critical points if $1 / \beta<r<\beta, r \neq 1$.

Assuming $\varepsilon$ small enough, each critical point gives rise to a homoclinic orbit, though a neighborhood of the rays $\sqrt{I_{1}^{0} / I_{2}^{0}}=0,1 / \beta, 1, \beta, \infty$ have to be excluded.

In the next theorem we study a more general case (i.e. not assuming $f=\mathcal{O}_{2}$ ), and establish the number of transverse homoclinic orbits, in a much simpler way.

Theorem 5 Consider an arbitrary $f$ in the perturbation (30). Under generic conditions the coefficients of order 1 of $f$, for an open subset of actions $I^{0}=\left(I_{1}^{0}, I_{2}^{0}\right)$, and $\varepsilon$ small enough, there exist exactly 4 transverse homoclinic orbits.

Proof. Let

$$
f=a_{0}+a_{11} x_{1}+a_{12} y_{1}+a_{21} x_{2}+a_{22} y_{2}+\mathcal{O}_{2}\left(x_{1}, y_{1}, x_{2}, y_{2}\right) .
$$

Computing the Melnikov potential as in the proof of Theorem 4, we obtain

$$
L=4 a_{0}+2 \sqrt{2 I_{1}^{0}} \mathcal{J}_{\omega_{1}}\left(a_{11} \cos \theta_{1}+a_{12} \sin \theta_{1}\right)+2 \sqrt{2 I_{2}^{0}} \mathcal{J}_{\omega_{2}}\left(a_{21} \cos \theta_{2}+a_{22} \sin \theta_{2}\right)+\mathcal{O}\left(\left\|I^{0}\right\|\right),
$$

and it has 4 critical points provided $a_{11}^{2}+a_{12}^{2} \neq 0$ and $a_{21}^{2}+a_{22}^{2} \neq 0$ (compare with condition (LLL) in Section 4). Such critical points tend to constant as $\left(I_{1}^{0}, I_{2}^{0}\right) \rightarrow(0,0)$ (in this case, the constant is the same for all rays $I_{1}^{0} / I_{2}^{0}=$ const). 
Although the $\theta_{i}$-derivatives of $L$ tend to 0 as $\left(I_{1}^{0}, I_{2}^{0}\right) \rightarrow(0,0)$, this does not imply the persistence of the homoclinic loop $\Gamma$ for $\varepsilon \neq 0$, since the splitting of this loop may appear in the higher order terms in (27). In the same way, the 4 critical points predicted by the Melnikov potential may not give true homoclinic intersections if $\left(I_{1}^{0}, I_{2}^{0}\right)$ is very close to $(0,0)$ (to be more precise, a neighborhood of the lines $I_{1}=0, I_{2}=0$, of width $\mathcal{O}\left(\varepsilon^{2}\right)$, has to be excluded from the open set in the statement of Theorem 5). Thus, the usual PoincaréMelnikov method, as introduced at the beginning of this section, does not apply in a small neighborhood of the loop.

\section{A first approximation for the splitting of the loop}

Finally, we show that the splitting of the loop $\Gamma$ can be detected with a modified version of the Poincaré-Melnikov method, that can be applied to the examples introduced in Section 1.1. Due to the degeneracy of the polar coordinates defined in (5), we develop the method directly in the original coordinates $z=\left(x_{1}, y_{1}, x_{2}, y_{2}, p, q\right)$.

We are going to obtain a first approximation for the splitting distance between the (global) invariant manifolds $W^{u}, W^{s}$ inherited from the initial loop $\Gamma$. The unstable manifold $W^{u}$ is given by a trajectory $z^{u}(t)=\left(x_{1}^{u}(t), y_{1}^{u}(t), x_{2}^{u}(t), y_{2}^{u}(t), p^{u}(t), q^{u}(t)\right)$ that tends to the singular point $O$ for $t \rightarrow-\infty$, and analogously with the stable manifold $W^{s}$ for $t \rightarrow \infty$. Both trajectories $z^{u}(t), z^{s}(t)$ are $\mathcal{O}(\varepsilon)$-perturbations of the loop $\Gamma$, given by $z_{0}(t)=\left(0,0,0,0, \dot{q}_{0}(t), q_{0}(t)\right)$ (notice that this is the limit of the homoclinic trajectory $(25)$ as $\left(I_{1}^{0}, I_{2}^{0}\right) \rightarrow(0,0)$, for any $\left.\left(\theta_{1}, \theta_{2}\right)\right)$.

To give a measure for the splitting distance between $W^{u}$ and $W^{s}$, we can restrict ourselves to the coordinates $\left(x_{1}, y_{1}, x_{2}, y_{2}\right)$. Indeed, the distance along $p$ is then determined (on a section $q=$ const) by the conservation of energy.

Theorem 6 Consider in the perturbation (30) an arbitrary $f$, written as in (36). Then, for $\varepsilon$ small enough the following approximations hold:

$$
\begin{aligned}
& x_{i}^{u}(t)-x_{i}^{s}(t)=-2 \varepsilon \mathcal{J}_{\omega_{i}}\left(a_{i 1} \cos \omega_{i} t-a_{i 2} \sin \omega_{i} t\right)+\mathcal{O}\left(\varepsilon^{2}\right), \\
& y_{i}^{u}(t)-y_{i}^{s}(t)=-2 \varepsilon \mathcal{J}_{\omega_{i}}\left(a_{i 1} \sin \omega_{i} t+a_{i 2} \cos \omega_{i} t\right)+\mathcal{O}\left(\varepsilon^{2}\right), \quad i=1,2 .
\end{aligned}
$$

Proof. We first consider the unstable manifold $W^{u}$. We write $x_{i}^{u}(t)=\varepsilon x_{i}^{u, *}(t)+\mathcal{O}\left(\varepsilon^{2}\right)$, $y_{i}^{u}(t)=\varepsilon y_{i}^{u, *}(t)+\mathcal{O}\left(\varepsilon^{2}\right)$, which satisfy the Hamiltonian equations

$$
\begin{aligned}
& \dot{x}_{i}^{u}=-\omega_{i} y_{i}^{u}+\mathcal{O}_{3}\left(x_{1}^{u}, y_{1}^{u}, x_{2}^{u}, y_{2}^{u}\right)-\varepsilon \frac{\partial H_{1}}{\partial x_{i}}\left(x_{1}^{u}, y_{1}^{u}, x_{2}^{u}, y_{2}^{u}, p^{u}, q^{u}\right), \\
& \dot{y}_{i}^{u}=\omega_{i} x_{i}^{u}+\mathcal{O}_{3}\left(x_{1}^{u}, y_{1}^{u}, x_{2}^{u}, y_{2}^{u}\right)+\varepsilon \frac{\partial H_{1}}{\partial y_{i}}\left(x_{1}^{u}, y_{1}^{u}, x_{2}^{u}, y_{2}^{u}, p^{u}, q^{u}\right) .
\end{aligned}
$$

Taking the $\mathcal{O}(\varepsilon)$-terms in these equations, we obtain that $x_{i}^{u, *}(t), y_{i}^{u, *}(t)$ satisfy the linear equations

$$
\dot{x}_{i}^{u, *}=-\omega_{i} y_{i}^{u, *}-\frac{\partial H_{1}}{\partial x_{i}}\left(0,0,0,0, \dot{q}_{0}(t), q_{0}(t)\right), \quad \dot{y}_{i}^{u, *}=\omega_{i} x_{i}^{u, *}+\frac{\partial H_{1}}{\partial y_{i}}\left(0,0,0,0, \dot{q}_{0}(t), q_{0}(t)\right) .
$$


Then, from the variation-of-constants formula we get the expressions

$$
x_{i}^{u, *}(t)=g_{i 1}^{u}(t) \cos \omega_{i} t-g_{i 2}^{u}(t) \sin \omega_{i} t, \quad y_{i}^{u, *}(t)=g_{i 1}^{u}(t) \sin \omega_{i} t+g_{i 2}^{u}(t) \cos \omega_{i} t,
$$

where

$$
\begin{aligned}
& g_{i 1}^{u}(t)=\int_{-\infty}^{t}\left(-\cos \omega_{i} s \cdot \frac{\partial H_{1}}{\partial x_{i}}\left(z_{0}(s)\right)+\sin \omega_{i} s \cdot \frac{\partial H_{1}}{\partial y_{i}}\left(z_{0}(s)\right)\right) d s \\
& g_{i 2}^{u}(t)=\int_{-\infty}^{t}\left(\sin \omega_{i} s \cdot \frac{\partial H_{1}}{\partial x_{i}}\left(z_{0}(s)\right)+\cos \omega_{i} s \cdot \frac{\partial H_{1}}{\partial y_{i}}\left(z_{0}(s)\right)\right) d s
\end{aligned}
$$

Using equality (31), we see that

$$
\frac{\partial H_{1}}{\partial x_{i}}\left(z_{0}(s)\right)=-\frac{2 a_{i 1}}{\cosh ^{2} t}, \quad \frac{\partial H_{1}}{\partial y_{i}}\left(z_{0}(s)\right)=-\frac{2 a_{i 2}}{\cosh ^{2} t},
$$

and this implies that the integrals (40-41) are absolutely convergent.

We can proceed in a similar way for the stable manifold $W^{s}$, and obtain expressions analogous to $(39-41)$, but now $\int_{-\infty}^{t}$ is replaced by $-\int_{t}^{\infty}$. Their difference is given at first order by

$x_{i}^{u, *}(t)-x_{i}^{s, *}(t)=\triangle g_{i 1} \cos \omega_{i} t-\triangle g_{i 2} \sin \omega_{i} t, \quad y_{i}^{u, *}(t)-y_{i}^{s, *}(t)=\triangle g_{i 1} \sin \omega_{i} t+\triangle g_{i 2} \cos \omega_{i} t$, with $\triangle g_{i j}$ as in (40-41), but now with $\int_{-\infty}^{\infty}$. Computing the integrals as in (32), we obtain $\triangle g_{i j}=-2 a_{i j} \mathcal{J}_{\omega_{i}}$, and this gives the first order approximations stated in (37-38).

Remarks 4 1. In this approach, we have measured the splitting along the coordinates $\left(x_{1}, y_{1}, x_{2}, y_{2}\right)$, which are not first integrals of the unperturbed Hamiltonian $H_{0}$ (unlike the approach followed in (29)). But the fact that, in $H_{0}$, the variation of such coordinates can be described by linear differential equations allowed us to obtain a first approximation analogous to the Melnikov function also in this case.

2. As a consequence of Theorem 6 , the distance between $W^{s}$ and $W^{u}$ along the first integrals $\xi_{i}=\left(x_{i}^{2}+y_{i}^{2}\right) / 2$ is $\mathcal{O}\left(\varepsilon^{2}\right)$. This explains why the splitting of the loop $\Gamma$ (as well as for tori close to $\Gamma$ ) could not be detected from the Melnikov approximation considered in Theorem 5.

3. The loop is not preserved if at least one of the coefficients $a_{i j}$ does not vanish. This agrees with the fact that the set $\mathcal{L}$ of Hamiltonians with homoclinic loops has codimension 4 , as explained at the beginning of Section 4.

\section{Acknowledgments}

This work has been supported in part by the INTAS grant 00-221. O.K. and L.L. also acknowledge a partial support of CRDF (award RU-M1-2583-MO-04) and Russian Foundation of Basic Research (grant 04-01-00483), L.L. acknowledges a support from the program "Universities of Russia" (project No. 1905). A.D. and P.G. were also partially supported by the Catalan grant 2001SGR-70, and the MCyT-FEDER grant BFM2003-9504. 


\section{References}

[1] R. Abraham and J. E. Marsden. Foundations of mechanics. Benjamin/Cummings, Reading, Mass., 1978. Second edition.

[2] V. I. Arnol'd. Mathematical methods of classical mechanics, volume 60 of Graduate Texts in Mathematics. Springer-Verlag, New York, second edition, 1989.

[3] J. Carr. Applications of centre manifold theory, volume 35 of Applied Mathematical Sciences. Springer-Verlag, New York, 1981.

[4] A. Delshams and P. Gutiérrez. Splitting potential and the Poincaré-Melnikov method for whiskered tori in Hamiltonian systems. J. Nonlinear Sci., 10(4):433-476, 2000.

[5] L. H. Eliasson. Normal forms for Hamiltonian systems with Poisson commuting integralselliptic case. Comment. Math. Helv., 65(1):4-35, 1990.

[6] S. M. Graff. On the conservation of hyperbolic invariant tori for Hamiltonian systems. $J$. Differential Equations, 15:1-69, 1974.

[7] M. W. Hirsch, C. C. Pugh, and M. Shub. Invariant manifolds. Springer-Verlag, Berlin, 1977. Lecture Notes in Mathematics, Vol. 583.

[8] L. M. Lerman. Isoenergetical structure of integrable Hamiltonian systems in an extended neighborhood of a simple singular point: three degrees of freedom. In L. Lerman, G. Polotovskil, and L. Shilnikov, editors, Methods of qualitative theory of differential equations and related topics, Supplement, volume 200 of Amer. Math. Soc. Transl. Ser. 2, pages 219-242. Amer. Math. Soc., Providence, RI, 2000. Advances in the Mathematical Sciences, 48.

[9] L. M. Lerman and Ya. L. Umanskiy. Four-dimensional integrable Hamiltonian systems with simple singular points (topological aspects), volume 176 of Translations of Mathematical Monographs. American Mathematical Society, Providence, RI, 1998.

[10] D. McDuff and D. Salamon. Introduction to symplectic topology. Oxford Mathematical Monographs. Oxford University Press, New York, second edition, 1998.

[11] J. Milnor. Morse theory. Annals of Mathematics Studies, No. 51. Princeton University Press, Princeton, N.J., 1963.

[12] J. Moser. The analytic invariants of an area-preserving mapping near a hyperbolic fixed point. Comm. Pure Appl. Math., 9:673-692, 1956.

[13] J. Pöschel. Integrability of Hamiltonian systems on Cantor sets. Comm. Pure Appl. Math., $35(5): 653-696,1982$.

[14] I. R. Shafarevich. Basic algebraic geometry. 1. Springer-Verlag, Berlin, second edition, 1994. Varieties in projective space.

[15] D. V. Treshev. Hyperbolic tori and asymptotic surfaces in Hamiltonian systems. Russian J. Math. Phys., 2(1):93-110, 1994.

[16] J. Vey. Sur certains systèmes dynamiques séparables. Amer. J. Math., 100(3):591-614, 1978. 